Canadian

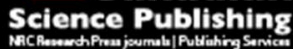

Canadian Geotechnical Journal Revue canadienne de géotechnique

\title{
Interpolating spatially varying soil property values from sparse data for facilitating characteristic value selection
}

\begin{tabular}{|r|l|}
\hline Journal: & Canadian Geotechnical Journal \\
\hline Manuscript ID & cgj-2017-0219.R1 \\
\hline Danuscript Type: & Article \\
\hline Complete List of Authors: & $\begin{array}{l}\text { Zhao, Tengyuan; City University of Hong Kong, Architecture and civil } \\
\text { engineering } \\
\text { Montoya-Noguera, Silvana; City University of Hong Kong } \\
\text { Phoon, Kok-Kwang; National University of Singapore, Department of Civil \& } \\
\text { Environmental Engineering } \\
\text { Wang, Yu; City University of Hong Kong, Dept of Civil and Architectural } \\
\text { Engineering }\end{array}$ \\
\hline Keyword: & $\begin{array}{l}\text { Reliability-based design, Bayesian compressive sampling, compressive } \\
\text { sensing, sparse measurement data, site investigation }\end{array}$ \\
\hline \multicolumn{2}{|c}{} \\
\hline
\end{tabular}




\section{$1 \quad$ Interpolating spatially varying soil property values from sparse}

\section{data for facilitating characteristic value selection}

$4 \quad$ Tengyuan Zhao ${ }^{1}$, Silvana Montoya-Noguera ${ }^{2}$, Kok-Kwang Phoon ${ }^{3}$ and Yu Wang ${ }^{4}$

\section{5}

$6{ }^{1}$ PhD Student, Department of Architecture and Civil Engineering, City University of Hong

$7 \quad$ Kong, Tat Chee Avenue, Kowloon, Hong Kong

$8{ }^{2}$ Research Associate, Department of Architecture and Civil Engineering, City University of

9 Hong Kong, Tat Chee Avenue, Kowloon, Hong Kong

$10{ }^{3}$ Distinguished Professor, Department of Civil and Environmental Engineering, National

11 University of Singapore, Singapore

$12{ }^{4}$ Associate Professor, Department of Architecture and Civil Engineering, City University of

13 Hong Kong, Tat Chee Avenue, Kowloon, Hong Kong. (Tel): 852-3442-7605 (Fax):852-

14 3442-0427 (Email): yuwang@cityu.edu.hk (Corresponding author) 


\section{Abstract}

16 Limit state design, incorporated into many recent geotechnical design codes, introduces the 17 application of partial or resistance factors to selected characteristic values. Partial or 18 resistance factors are usually set by national standard organizations, while characteristic 19 values of geotechnical parameters are selected by engineers, often based on sparse 20 measurement data combined with subjective engineering experience and judgment. Due to 21 this subjective selection and individual judgment, the characteristic value derived by different 22 engineers from the same dataset may vary greatly, especially when the test data contain 23 significant variability. To address this issue, a new method based on Bayesian compressive 24 sampling (BCS) is proposed in this study. BCS is able to reconstruct a high-resolution 25 geotechnical property profile from sparse measurement data and quantify the uncertainty, e.g. 26 confidence interval (CI) associated with the interpreted profile. The quantified uncertainty in 27 the BCS has a clear statistical meaning: the corresponding confidence level for a CI from the 28 BCS is the expected coverage proportion (i.e. fraction) of the complete profile that falls 29 within the CI, if all data points along depth can be measured to provide the complete profile.

30 This statistical meaning can be used to facilitate objective determination of characteristic 31 values for geotechnical properties.

33 Keywords: Reliability-based design; Bayesian compressive sampling; compressive sensing; 34 sparse measurement data; site investigation 


\section{Introduction}

36 Limit state design methods have been recently incorporated into many geotechnical codes of

37 practice throughout the world, e.g. Eurocode 7 (CEN 2004), AASHTO Bridge Code

38 (AASHTO 1998), and Canadian Highway Bridge Design Code (CHBDC 2014), among

39 others. To achieve a specific target reliability level, the design value is determined by

40 dividing the characteristic strength values by partial factors (e.g. Meyerhof 1995; Fenton and

41 Naghibi 2011; Reddy and Stuedlein 2017) or multiplying the characteristic resistance values

42 by resistance factors. Partial or resistance factors are usually set by national standard

43 organizations and are used to achieve a target level of reliability or safety (although explicit

44 reliability calibration may not be conducted); while the characteristic values of geotechnical

45 parameters are selected by geotechnical engineers. In engineering practice, these

46 characteristic values are often selected based on a limited number of test results, therefore

47 engineering judgment and previous relevant experience are frequently used to select the

48 characteristic values (e.g. Orr 2017).

49 Because of this subjective selection and individual judgment, the characteristic values,

50 derived by different geotechnical engineers from the same dataset, may vary greatly,

51 especially when the test data are scarce or contain significant variability. For example, Bond

52 and Harris (2008) presented three case studies in which about one hundred engineers were

53 asked to select the characteristic values on the basis of Eurocode 7 from the same set of test

54 data. The case studies dealt with different types of data (SPT blow counts, field vane tests and

55 triaxial tests), different soil types (clays and gravels) and different number of data points

56 (from 25 to above 100 points for profiles of 10 to $30 \mathrm{~m}$ deep). The selected characteristic

57 values varied greatly, and the maximum characteristic value obtained was about 3 to 5 times

58 greater than the minimum one. Orr (2017) suggested that more guidance is needed to select 
59 characteristic values in an objective manner to reduce and properly account for this broad 60 range of interpretation.

61 Statistical analyses of laboratory and in-situ test results to determine geotechnical 62 parameters for reliability-based design applications have been broadly discussed and 63 recommended in the literature (e.g. Vanmarcke 1977; Phoon and Kulhawy 1999; Baecher and 64 Christian 2003; Fenton and Griffiths 2008; Becker 2010; Gong et al. 2014; Li et al. 2016; 65 Phoon et al. 2016). Although statistical methods are explicitly recommended in some design 66 guides (e.g. Det Norske Veritas 2010), currently the use of such analyses has not been 67 included in some existing design codes, such as Eurocode 7, partially because of the limited number of site specific measurement data and the inherent variability encountered in natural soil deposits (Orr 2017). Furthermore, most of the available statistically based methods for determining the characteristic values of geotechnical parameters focus on point statistics (e.g. mean and coefficient of variation for a previously defined homogeneous soil layer) and hence 72 ignore the spatially varying pattern of soil properties (e.g. Cao and Wang 2014; Wang et al. 73 2016a; Wang and Aladejare 2016; Wang and Cao 2013).

74 This paper aims to address these two issues, limited data and inherent spatial variability, and to provide a statistical procedure for an objective determination of characteristic value from spatially varying but sparsely measured data. It uses compressive sampling theory to reconstruct the best estimate of a soil property profile from sparse measurement data points (Wang and Zhao 2016) and Bayesian theory to estimate the 79 statistical uncertainty associated with the interpreted profile (Wang and Zhao 2017). The use 80 of the Bayesian framework acknowledges the critical role of engineering judgment but 81 reduces the subjective interpretation uncertainty by quantitatively representing it as prior 82 knowledge (e.g. Cao, Wang and Li 2016; Vick 2002; Wang and Aladejare 2015; Wang et al. 83 2016b). 
This paper first presents an interpretation of the statistical meaning of the confidence interval for random field data. Then it reviews the formulation of BCS and uses it to provide average and confidence interval profiles, given only sparsely measured but spatially varying geotechnical data. Note that the measurement data of soil properties obtained in geotechnical engineering are usually sparse and limited, particularly for small or medium sized projects. An important question when interpreting sparse data in geotechnical practice is: how does the profile interpreted from sparse data compare with the measured profile, if it is possible to measure the geotechnical data with a small interval and a high resolution? This paper shows that the confidence interval $(\mathrm{CI})$ profile quantified in BCS has a clear statistical meaning, which may be used to address this question and to facilitate determination of characteristic values in engineering practice. For illustration, the proposed BCS procedure is applied to a real case of CPT data and the selection of the characteristic value of effective friction angle.

This paper addresses the characteristic value only from a purely statistical perspective, although characteristic value may be related to the extent of failure zone governing the behavior of the structure at the limit state. For example, some researchers have argued that the characteristic value is related to the concept of a mobilized strength along the critical slip surface (Ching and Phoon 2013a, 2013b; Ching et al. 2014, 2016a) or a mobilized modulus over a domain influenced by the structure at the limit state (Ching et al. 2016b). As the limit state of a geo-structure is problem dependent, and a realistic assessment of the characteristic value in the context of spatial variability where non-classical failure mechanisms can emerge

104 is less straightforward, the extent of failure zone governing the behavior of the structure at the limit state is not considered in this paper.

106

\section{Coverage proportion of confidence interval profiles}


108 The confidence interval (CI), which may be used to quantify uncertainty, is more informative

109 than simply reporting a point estimate (e.g. Phoon and Ching 2014). CI is an interval

110 estimation of a parameter of interest which gives a confidence level that the true parameter

111 falls within the estimated CI. To evaluate the confidence interval, analytical equations can be

112 used when the distribution of the data is known. For example, for normally distributed data

113 with a known mean $(\mu)$ and standard deviation $(\sigma)$, the CI for a confidence level $\alpha$, denoted as

$114 \quad \mathrm{CI}_{\alpha}$, is expressed as:

115

$$
\mathrm{CI}_{\alpha}=\mu \pm z_{(1-\alpha) / 2} \sigma
$$

117 where $\left.z_{(1-\alpha) / 2}=-\Phi^{-1}[(1-\alpha) / 2)\right]$ and $\Phi^{-1}(\cdot)$ is the inverse standard normal cumulative

118 distribution function. Note that the lower bound of Equation (1) or its variants (i.e. $\mu$ minus a

119 factored $\sigma$ ) has been proposed in literature, e.g. Schneider and Schneider (2013) and Orr

120 (2017) as characteristic value at a given depth or for a homogeneous soil layer in Eurocode 7.

121 For spatially varying data, such as random field samples (RFSs), the values vary along a

122 spatial dimension (e.g. depth), hence the mean and $\mathrm{CI}_{\alpha}$ also vary along this spatial dimension,

123 e.g. profiles varying with depth. For a given random field with known $\mu$ and $\sigma, \mathrm{CI}_{\alpha}$ profiles

124 (i.e. variations of $\mathrm{CI}_{\alpha}$ with depth) can be generated analytically by applying Equation (1) to

125 different depths. For example, the $\mathrm{CI}_{90 \%}$ profiles can be obtained by substituting $\alpha=90 \%$ to

126 Equation (1) for different depths of interest.

127

$128 \boldsymbol{C P}_{\alpha}$ of $\mathrm{CI}_{\alpha}$ profiles for random field data 
129 The coverage proportion $\left(\mathrm{CP}_{\alpha}\right)$ of a soil property profile $\boldsymbol{f}$, e.g. a RFS, that falls within a $\mathrm{CI}_{\alpha}$ 130 profile with a confidence level $(\alpha)$ is defined as (e.g. Marra and Wood 2012; Nychka 1988;

131 Wahba 1983):

132

$$
\mathrm{CP}_{\alpha}=\frac{1}{\mathrm{~N}} \sum_{k=1}^{\mathrm{N}}\left[\mathrm{I}\left(f_{k} \in \mathrm{CI}_{\alpha}\right)\right]
$$

134 where $\mathrm{N}$ is the total number of data points in the soil property profile $\boldsymbol{f}$, and $\mathrm{I}(\cdot)$ is the 135 indicator function. $\mathrm{I}(\cdot)$ equals to unity if a data point $f_{k}(k=1,2, \ldots, \mathrm{N})$ is within the upper 136 and lower bounds of $\mathrm{CI}_{\alpha}$, and otherwise, it is zero. Note that the expected value of $\mathrm{CP}_{\alpha}$ is 137 equal to $\alpha$ (Wahba 1983). For example, $\mathrm{CI}_{95 \%}$ implies that $95 \%$ of all data points are expected 138 to fall within the upper and lower bounds given by $\mathrm{CI}_{95 \%}$, i.e. $\mathrm{CP}_{95 \%}=95 \%$. The coverage 139 proportion has been evaluated for $\mathrm{CI}_{\alpha}$ profiles obtained from smoothing functions (e.g. 140 Nychka 1988; Wahba 1983) and generalized additive models (e.g. Marra and Wood 2012). In 141 this section, the procedure to evaluate the $\mathrm{CP}_{\alpha}$ of a RFS that falls within a $\mathrm{CI}_{\alpha}$ profile is firstly explained. Then the procedure is illustrated with simulated random field data. This section is meant to explain the definition and evaluation of $\mathrm{CP}_{\alpha}$, and it paves the way for the next section where $\mathrm{CP}_{\alpha}$ will be evaluated for the $\mathrm{CI}_{\alpha}$ profiles obtained from the $\mathrm{BCS}$ method with a limited number of measurement data as input.

146 Note that if the random field has no correlation (i.e. the data points over the depth are 147 independent), the probability distribution of $\mathrm{CP}_{\alpha}$ follows a binomial distribution because of 148 the indicator function in Equation (2) (e.g. De Veaux et al. 2014; Efron and Tibshirani 1993).

149 Hence, the average $\mathrm{CP}_{\alpha}$ is equal to $\alpha$, and the variance is equal to $\alpha(1-\alpha) / \mathrm{N}$. The shape of 150 the distribution of $\mathrm{CP}_{\alpha}$ is symmetric (i.e. zero skewness) for $\alpha=0.5$ and it is negatively skewed 
151 as $\alpha$ approaches 1 (De Veaux et al. 2014). The distribution of $\mathrm{CP}_{\alpha}$ tends to the normal

152 distribution as $\mathrm{N}$ increases and $\alpha$ approaches 0.5. De Veaux et al. (2014) suggest that a 153 normal distribution gives a good approximation of the binomial distribution if $\mathrm{N} \alpha \geq 10$ and $154 \mathrm{~N}(1-\alpha) \geq 10$.

156 Simulation of random field examples

157 For illustration, a 1D stationary Gaussian random field is used to represent a soil property X 158 profile. Random field samples (RFSs) are generated using a truncated Karhunen-Loève (KL) 159 expansion. Truncated KL expansion has been increasingly studied and used in simulating 1D 160 random processes in recent years (e.g. Zhang and Ellingwood 1994; Phoon et al. 2002; Phoon 161 et al. 2005; Li et al. 2014). The following parameters were used: mean $\mu_{X}=30$, standard 162 deviation $\sigma_{X}=2$ and an exponential correlation function, i.e. $\rho_{i, j}=\exp \left(-\frac{2\left|d_{X_{i}}-d_{X_{j}}\right|}{\lambda_{c}}\right)$, 163 where $d_{X_{i}}$ and $d_{X_{j}}$ are the depths of two $\mathrm{X}$ data points $\mathrm{X}_{i}$ and $\mathrm{X}_{j}$, respectively, and $\lambda_{c}$ is the 164 correlation length taken as $2 \mathrm{~m}$ in this example. The soil layer thickness (h) is taken as $20.44 \mathrm{~m}$ 165 and the profile has a resolution of $0.04 \mathrm{~m}$. Hence there are $\mathrm{N}=512$ points for each RFS. Note 166 that only one homogeneous soil layer (rather than several soil layers) with a thickness of $16720.44 \mathrm{~m}$ is considered in this illustrative example. Stratification therefore is not needed in this 168 example. For the truncated KL expansion, $200 \mathrm{KL}$ terms are used. Note that 200 terms are 169 able to preserve $98.2 \%$ of the total variance of the random field in terms of the sum of 200 170 eigenvalues and the sum of all eigenvalues in this example. Therefore, it accurately 171 represents the prescribed random field (e.g. Phoon et al. 2002). The number of RFS $\left(\mathrm{N}_{\mathrm{s}}\right)$ 172 generated is 1000. All RFSs generated are shown in Figure 1 in light gray. Additionally, the 173 profile of the mean values, evaluated as the average of all $\mathrm{N}_{\mathrm{s}}$ values at each depth, is also 174 shown. 
176 Probability distribution of $\mathrm{CP}_{\alpha}$ for $\mathrm{CI}_{\alpha}$ profiles

177 The $\mathrm{CI}_{\alpha}$ profiles over depth are constructed using Equation (1) with a given confidence level

$178 \alpha$. For example, given $\alpha=95 \%$, the $\mathrm{CI}_{95 \%}$ profiles are obtained with the $2.5^{\text {th }}$ and $97.5^{\text {th }}$

179 percentiles from Equation (1) and are shown in Figure 1 by two dotted lines. In each subplot

180 of this figure, one RFS is shown in black solid line to illustrate the evaluation of $\mathrm{CP}_{95 \%}$. For

181 each one of these samples, the coverage proportion of the RFS profile that is within the $\mathrm{CI}_{95 \%}$

182 profiles (i.e. between the two dotted lines in Figure 1) is evaluated. The $\mathrm{CP}_{95 \%}$ values for the

183 three RFSs presented in Figure 1 are $94.5 \%, 94.9 \%$ and $96.1 \%$, respectively.

184 The $\mathrm{CP}_{\alpha}$ values are evaluated for all $\mathrm{N}_{\mathrm{s}}=1000$ RFSs and for different $\alpha$ values ranging

185 from $50 \%$ to $95 \%$. Statistical analysis is performed for the $\mathrm{CP}_{\alpha}$ values obtained, and the

186 results are shown as box-and-whiskers plots for different $\alpha$ values in Figure $2 \mathrm{~b}$. The box is

187 constructed with the inter-quartile range, IQR $=25 \%-75 \%$ percentiles, and the whiskers

188 show the minimum and maximum values within $1.5 \mathrm{IQR}$. The maximum and minimum $\mathrm{CP}_{\alpha}$

189 values among all $\mathrm{N}_{\mathrm{s}}$ RFSs are shown by crosses in Figure 2, and the mean $\mathrm{CP}_{\alpha}$ values are

190 shown with circles. Figure 2 also includes a 1:1 line in each subplot. The mean $\mathrm{CP}_{\alpha}$ values for

191 the $\alpha$ value varying from $50 \%$ to $95 \%$ all plot along the $1: 1$ line, and the average $\mathrm{CP}_{\alpha}$ is equal

192 to $\alpha$. The $\mathrm{CI}_{\alpha}$ profiles can be statistically interpreted as the upper and lower bounds of an

193 interval where the expected coverage proportion (i.e. fraction) of a RFS (i.e. a spatially

194 variable soil property X profile) that falls within the interval is $\alpha$.

195 Note that, although the expectation of $\mathrm{CP}_{\alpha}$ is $\alpha$, the $\mathrm{CP}_{\alpha}$ value for each RFS may vary

196 significantly, as shown in Figure 2. When the $\mathrm{CP}_{\alpha}$ values are greater than the $\alpha$ values, a

197 relatively large proportion of the RFS fall within the interval. In contrast, when the $\mathrm{CP}_{\alpha}$

198 values are smaller than the $\alpha$ values, a relatively large proportion of the RFS fall outside the 
$199 \mathrm{CI}_{\alpha}$ profiles. For example, for the worst case in Figure $2 \mathrm{~b}$, the smallest $\mathrm{CP}_{50 \%}$ value is close to $20020 \%$. This means that, besides the $50 \%$ expected, an additional $30 \%$ of the RFS falls outside 201 the $\mathrm{CI}_{50 \%}$ profiles. For all $\mathrm{N}_{\mathrm{s}}=1000$ RFSs, less than $3 \%$ of RFSs present a $\mathrm{CP}_{95 \%}$ lower than $20285.5 \%$ (i.e. a relative difference of $10 \%$ on the expected value). In contrast, for $\mathrm{CP}_{50 \%}$, about $20330 \%$ of RFSs are below $45 \%$ (i.e. the same $10 \%$ difference). As the confidence level 204 increases, the variability of the $\mathrm{CP}$ values decreases, as shown by the decreasing size of the 205 box and whiskers as the $\alpha$ value approaches unity. In addition, the mean and median $\mathrm{CP}_{\alpha}$ 206 values (shown with a line inside the box in Figure 2) are similar for $\alpha$ equal 50\%, but the 207 median $\mathrm{CP}_{\alpha}$ value is slightly larger than the mean $\mathrm{CP}_{\alpha}$ value for high $\alpha$ values. In other 208 words, the $\mathrm{CP}_{\alpha}$ results are symmetric, i.e. present zero skewness, for $\alpha$ close to 0.5 , but 209 develop a negative or left skewness as $\alpha$ approaches unity. This is similar to the effect of

210 small $\mathrm{N}$ values when the random field has no correlation and the $\mathrm{CP}_{\alpha}$ values follow a

211 binomial distribution (De Veaux et al. 2014).

212

213 Effect of different correlation length in random field

214 The same procedure as described previously was used for generating RFSs with various $\lambda_{c}$ 215 values. Figures $2 \mathrm{a}$ and $2 \mathrm{c}$ show the box-and-whiskers plots of the corresponding $\mathrm{CP}_{\alpha}$ results 216 for $\lambda_{c}=0.5 \mathrm{~m}$ and $5 \mathrm{~m}$, respectively. For all the cases, the mean $\mathrm{CP}_{\alpha}$ is equal to $\alpha$, although the 217 results present more variability for the case of $\lambda_{c}=5 \mathrm{~m}$. The variability of $\mathrm{CP}_{\alpha}$ increases as 218 the correlation length increases, as shown by the size of the box and the length of the 219 whiskers in Figure 2. Compared to the case of $\lambda_{c}=2 \mathrm{~m}$, the $\mathrm{CP}_{\alpha}$ results for the case of $\lambda_{c}=$ $2200.5 \mathrm{~m}$ are more concentrated around $\alpha$. The decrease in the $\mathrm{CP}_{\alpha}$ variability as $\alpha$ increases is 221 less visible for the case of $\lambda_{c}=0.5 \mathrm{~m}$. Also, for the case of $\lambda_{c}=0.5 \mathrm{~m}$, the median $\mathrm{CP}_{\alpha}$ is also 222 equal to $\alpha$, and all $\mathrm{CP}_{\alpha}$ distributions appear to be symmetric. In contrast, for the case of $\lambda_{c}=$ 
$2235 \mathrm{~m}$ shown in Figure 2c, the variability of $\mathrm{CP}_{\alpha}$ increases, and distributions are markedly 224 skewed for high $\alpha$ values. It can be seen that, for $\alpha$ greater than 0.7 , the median $\mathrm{CP}_{\alpha}$ values are greater than $\alpha$. Thus more than $50 \%$ of RFSs have a $\mathrm{CP}_{\alpha}$ equal to or greater than $\alpha$.

A total of eight different $\lambda_{c}$ values were tested ranging from 0.1 to $10 \mathrm{~m}$. This range of correlation length is consistent with those of geotechnical properties reported in literature (e.g. Phoon and Kulhawy 1999). The box plots of the $\mathrm{CP}_{\alpha}$ results are shown in Figure 3a for three $\alpha$ values: $50 \%, 80 \%$ and $95 \%$. For all results, the $\mathrm{CP}_{\alpha}$ mean values are equal to the corresponding $\alpha$ values, independent of either $\lambda_{c}$ or $\alpha$. However, the median values are greater than the mean values for high confidence levels, and the difference between mean and median values increases with the correlation length. Furthermore, the size of the boxes in Figure $3 \mathrm{a}$ increases with $\lambda_{c}$, suggesting that the variability of the $\mathrm{CP}_{\alpha}$ results increases with $\lambda_{c}$.

To visualize the effect of the correlation length and the confidence level on the variation of $\mathrm{CP}_{\alpha}$ results, Figure $3 \mathrm{~b}$ shows the standard deviation evaluated for $\mathrm{CP}_{\alpha}\left(\sigma_{\mathrm{CP} \alpha}\right)$ as a function of $\alpha$. When there is no correlation in the random field, the standard deviation is evaluated as $\sigma_{\mathrm{CP} \alpha}=\sqrt{\alpha(1-\alpha) / \mathrm{N}}$, as for the binomial distribution, and shown by a solid line in Figure 3b. A random field with no correlation was tested and the results obtained for $\sigma_{\mathrm{CP} \alpha}$ agree well with the analytical solution. When there is no correlation, the $\sigma_{\mathrm{CP} \alpha}$ reaches its maximum for $\alpha=0.5$. However, as $\lambda_{c}$ increases, the maximum value of $\sigma_{\mathrm{CP} \alpha}$ occurs at a relatively large $\alpha$ value. For $\lambda_{c}$ equal to $10 \mathrm{~m}$, a maximum $\sigma_{\mathrm{CP} \alpha}$ of about 0.18 is found at $\alpha=$ 0.65. In general, the $\sigma_{\mathrm{CP} \alpha}$ increases as $\lambda_{c}$ value increases. But this increase is more

244 pronounced for relatively small $\alpha$ values. For $\alpha=0.95$, the $\sigma_{\mathrm{CP} \alpha}$ is quite small, even for high correlation lengths because the upper bound of $\mathrm{CP}_{95 \%}=1$ is reached in many cases. 
247 random field with known parameters. In geotechnical engineering practice, if a soil property

248 profile is represented by a random field, its random field parameters, such as mean, standard

249 deviation and correlation function, are often difficult to estimate from measurement data,

250 especially the last two parameters. This is in part because the measurement data of soil

251 properties are usually limited and sparse. To address this difficulty, a Bayesian compressive

252 sampling method has been recently developed to statistically interpret the sparse

253 measurement data points for providing the best estimate and $\mathrm{CI}_{\alpha}$ profiles of the soil properties

254 (Wang and Zhao 2017), as briefly reviewed in the next section.

255

256 Review of Bayesian compressive sampling (BCS)

257 Bayesian compressive sampling (BCS) is a coupling of compressive sampling or sensing

258 (CS) and the Bayesian method to reconstruct the average and standard deviation profiles of a

259 soil property profile from only partial information of the profile, i.e. sparse measurement data

260 points (e.g. Ji et al. 2008; Wang and Zhao 2017). CS, mainly applied in electrical engineering

261 and computer science, exploits sparsity, or compressibility, in many real-world signals (e.g.

262 Candès et al. 2006; Candès and Wakin 2008). A signal, denoted as a column vector $\boldsymbol{f}$ with a

263 length of $\mathrm{N}$, is defined as the variation of a physical quantity with time or space.

264 "Compressibility" means that a signal $\boldsymbol{f}$ can be represented concisely as a weighted

265 summation of a proper type of basis functions, such as wavelet functions. In the mathematical

266 formulation of CS, $\boldsymbol{f}$ is expressed as follows:

267

$$
\boldsymbol{f}=\mathbf{B} \boldsymbol{\omega}
$$


269 where $\mathrm{B}$ is a $\mathrm{N} \times \mathrm{N}$ orthonormal matrix composed of columns of pre-specified basis functions,

270 and $\boldsymbol{\omega}$ is the corresponding weight coefficient vector with a length of $\mathrm{N}$. Because of the 271 compressibility of signals, most entries in $\boldsymbol{\omega}$ are near to zero. Thus, $\boldsymbol{f}$ can be reconstructed by

272 identifying and estimating the weight coefficients with significant value using the sparse 273 measurement data vector $\boldsymbol{y}$ that has a length of $\mathrm{M}$, where $\mathrm{M}<\mathrm{N}$, as follows:

$$
\boldsymbol{y}=\boldsymbol{\Psi} \boldsymbol{f}=\mathbf{A \omega}
$$

where $\boldsymbol{\Psi}$ is a $\mathrm{M} \times \mathrm{N}$ matrix and represents the locations of components $\boldsymbol{y}$ in $\boldsymbol{f} . \mathbf{A}=\boldsymbol{\Psi} \mathbf{B}$ is also a $\mathrm{M} \times \mathrm{N}$ matrix (Wang and Zhao 2016). Exploiting sparsity, the resulting underdetermined system of linear equations, i.e. Equation (4), can be solved by various existing efficient algorithms (e.g. Foucart and Rauhut 2013). For example, Wang and Zhao (2017) used a Bayesian method to statistically reconstruct the signal $\widehat{\boldsymbol{f}}$, which is an approximation of $\boldsymbol{f}$.

281 Mathematically, $\hat{\boldsymbol{f}}$ is defined by:

$$
\widehat{\boldsymbol{f}}=\mathbf{B} \boldsymbol{\omega}_{s}
$$

where $\boldsymbol{\omega}_{\boldsymbol{s}}$ is the approximate weight coefficient vector with a length of $\mathrm{N}$, and all components are set to zero except for the $\mathrm{S}$ non-trivial components $(\mathrm{S}<<\mathrm{N})$. Following the Bayesian framework (Wang and Zhao 2017), the posterior marginal distribution of $\boldsymbol{\omega}_{\boldsymbol{s}}$ derived from $\boldsymbol{y}$ follows a multivariate Student $t$ distribution with a degree of freedom equal to $2 c_{n}$ and a scale 
matrix of $\left(d_{n} / c_{n}\right) \mathbf{H}$. The mean and covariance matrix of $\boldsymbol{\omega}_{\boldsymbol{s}}$ (i.e. $\mu_{\boldsymbol{\omega}_{\boldsymbol{s}}}$ and $\mathbf{C O V}_{\boldsymbol{\omega}_{\boldsymbol{s}}}$, respectively) are expressed as:

$$
\begin{gathered}
\mu_{\omega_{s}}=\mathbf{H A}^{\mathrm{T}} \boldsymbol{y} \\
\operatorname{cov}_{\omega_{s}}=\frac{d_{n} \mathbf{H}}{c_{n}-1}
\end{gathered}
$$

where $\mathbf{H}=\left(\mathbf{A}^{\mathbf{T}} \mathbf{A}+\mathbf{D}\right)^{-1}, c_{n}=\mathrm{M} / 2+c_{0}$ and $d_{n}=\left(\boldsymbol{y}^{\boldsymbol{T}} \boldsymbol{y}-\mu_{\boldsymbol{\omega}_{s}}^{\mathrm{T}} \mathbf{H}^{-\mathbf{1}} \mu_{\boldsymbol{\omega}_{s}}\right) / 2+d_{0} \cdot c_{0}$ and $d_{0}$

293 are small non-negative constants, e.g. $c_{0}=d_{0}=10^{-4}$, $\mathbf{D}$ is a $\mathrm{N} \times \mathrm{N}$ diagonal matrix with

294 components $\mathrm{D}_{i, i}=\alpha_{i}$ and $\alpha_{i}$ are unknown non-negative coefficients. Note that Equation (6)

295 only depends on $\alpha_{i}$ and requires an iterative algorithm (e.g. the maximum likelihood

296 estimation) to obtain the most probable value of $\alpha_{i}$. Only the $\alpha_{i}$ values corresponding to the $\mathrm{S}$

$297(\mathrm{~S}<<\mathrm{N})$ non-trivial coefficients in $\omega_{s}$ need to be estimated in BCS, hence bypassing the

298 possible problem caused by high dimensionality. The number $\mathrm{S}$ of coefficients needed is

299 obtained by an iteration procedure using cosine similarity (Wang and Zhao 2017).

$300 \quad$ Because $\boldsymbol{\omega}_{\boldsymbol{s}}$ follows a multivariate Student $\mathrm{t}$ distribution and Equation (5), $\hat{\boldsymbol{f}}$ is also 301 derived as a random vector following a multivariate Student $t$ distribution (e.g. Ang and Tang 302 2007; Fenton and Griffiths 2008), with $2 c_{n}$ degree of freedom, mean $\mu_{\hat{f}}$ and scale matrix $\left(d_{n} / c_{n}\right) \mathbf{B H B} \mathbf{B}^{\mathrm{T}}$. The mean and covariance of $\hat{\boldsymbol{f}}$ (i.e. $\mu_{\hat{\boldsymbol{f}}}$ and $\mathbf{C O V}_{\hat{\boldsymbol{f}}}$, respectively) are derived as: 


$$
\begin{gathered}
\mu_{\hat{f}}=\mathbf{B} \mu_{\omega_{s}} \\
\operatorname{CoV}_{\hat{f}}=\mathbf{B C O V}_{\omega_{s}} \mathbf{B}^{\mathrm{T}}=\frac{d_{n}}{c_{n}-1} \mathbf{B H B}^{\mathrm{T}}
\end{gathered}
$$

306 The BCS procedure has been implemented by a package of user functions in MATLAB

307 (Mathworks, 2016). Only the sparse measurement data from site characterization are required 308 to obtain the mean and CI profiles of soil properties of interest.

In the following section, the BCS method is used to provide the best estimate of a

310 complete soil property profile from sparse measurement data and construct the associated CI

311 profiles. A statistical meaning of the CI profiles obtained from BCS is proposed: the 312 corresponding confidence level for a $\mathrm{CI}$ profile from BCS is the expected coverage 313 proportion (i.e. fraction) of the complete profile that falls within the CI, if all data points

314 along depth can be measured to provide the complete profile. The statistical meaning of the

315 BCS CI profiles is similar to that of the CI profiles for random field data shown in the section 316 "Coverage proportion of confidence interval profiles". The statistical meaning of the BCS CI 317 profiles will be evaluated systematically in the next section.

\section{Coverage proportion of the BCS confidence interval profiles}

320 Construction of CI profiles obtained from BCS with sparse measurement data as input

321 As $\hat{\boldsymbol{f}}$ follows a multivariate Student $t$ distribution (Wang and Zhao 2017), the upper and 322 lower bounds of the confidence interval are defined as (e.g. Taboga 2012): 


$$
\mathbf{C I}_{\alpha}=\mu_{\hat{f}} \pm t_{(1-\alpha) / 2,2 c_{n}} \sqrt{\left(2 c_{n}-2\right) / 2 c_{n}} \sqrt{\operatorname{diag}\left(\mathbf{C O V}_{\hat{f}}\right)}
$$

325 where $t_{(1-\alpha) / 2,2 c_{n}}$ is the Student $\mathrm{t}$ factor for a confidence level $\alpha$ and a degree of freedom

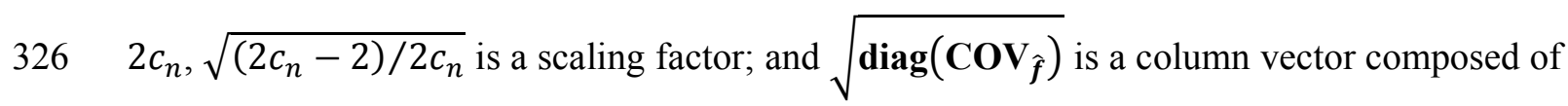

327 the square root of the diagonal elements of $\mathbf{C O V} \mathbf{V}_{\hat{\boldsymbol{f}}}$. Note that $\mathbf{C I}_{\boldsymbol{\alpha}}$ is composed of two column

328 vectors, which correspond to the upper and lower bounds of the two-tailed data distribution at

329 various depths (e.g. for $\alpha=90 \%$, the lower and upper bounds are the $5^{\text {th }}$ and $95^{\text {th }}$ percentiles, 330 respectively).

331 Consider, for example, using BCS to reconstruct the three RFSs shown in Figure 1 332 with only $M=20$ measurement data points from each RFS as input (i.e. the sparse 333 measurement data $\boldsymbol{y}$ ). Figure 4 shows the $M=20$ measurement data points by open circles. 334 The best estimate (i.e. mean) of the complete soil property X profile and $95 \%$ CI profiles are 335 shown in Figure 4 as dashed and dotted lines, respectively. Additionally, the original and 336 complete RFS profile is shown by a solid line in Figure 4 for comparison. Figure 4 shows 337 that, although the best estimate (i.e. the dashed line) does not go exactly through the 338 measurement data points (i.e. open circles), it follows a trend similar to that of these data 339 points and the original and complete RFS profiles. This suggests that the soil property X 340 profile reconstructed from BCS is consistent with the original variation of $\mathrm{X}$ with depth, even 341 when only a limited number of measurement data points (e.g. 20 from a total of 512 data 342 points) are used as input.

343 Some local variations of the original profile are not reconstructed in the best estimate 344 profile. This is because the number of measurement data is too limited (i.e. $\mathrm{M}=20 \ll \mathrm{N}=512$ ), 
345 hence the statistical uncertainty is quite significant. The statistical uncertainty can be 346 explicitly and objectively quantified by the covariance calculated in Equation (7) and the CI 347 profiles calculated with Equation (8). For example, the bounds of the $\mathrm{CI}_{95 \%}$ profiles are 348 shown in Figure 4 by two dotted lines. Similar to the CI profiles for random field data 349 discussed previously, the BCS CI profiles have a statistical meaning: the corresponding 350 confidence level for a BCS CI profile is the expected coverage proportion (i.e. fraction) of the 351 complete profile that falls within the CI, if all data points over depth can be measured to 352 provide the complete profile. In other words, although some details of the original profile are 353 not reflected by the best estimate from only 20 measured data points, on average around $95 \%$ 354 of all local variations of the original profile fall within the $\mathrm{CI}_{95} \%$ upper and lower bounds. For 355 example, the $\mathrm{CP}_{95 \%}$ values of the three original RFSs shown in Figure 4 are $94.9 \%, 94.1 \%$ 356 and $96.3 \%$, respectively. Hence, about $95 \%$ of the three original RFSs shown in Figure 4 fall 357 within the $\mathrm{CI}_{95 \%}$ upper and lower bounds. Recall that, in the subsection "Probability 358 distribution of $\mathrm{CP}_{\alpha}$ for $\mathrm{CI}_{\alpha}$ profiles", the similar $\mathrm{CP}_{95 \%}$ values evaluated for the full set of 359 random field data were $94.5 \%, 94.9 \%$ and $96.1 \%$, respectively. The difference is less than $1 \%$ 360 and quite minor.

\section{Probability distribution of $\mathrm{CP}_{\alpha}$ for $\mathrm{BCS} \mathrm{CI}_{\alpha}$ profiles}

363 To evaluate the probability distribution of $\mathrm{CP}_{\alpha}$ for the $\mathrm{BCS}_{\mathrm{CI}}$, the $\mathrm{BCS}$ method is used to

364 construct each of the $\mathrm{N}_{\mathrm{s}}=1000$ RFSs shown in Figure 1, using a limited number, e.g. $\mathrm{M}=20$, 365 of measurement data points from each RFS as input (i.e. $\boldsymbol{y}_{\mathrm{i}}, \mathrm{i}=1,2, \ldots, \mathrm{N}_{\mathrm{s}}$ ). This leads to a 366 total of 1000 best estimates and CI profiles obtained from BCS. Each of the 1000 RFSs is 367 used as the original and complete soil property $\mathrm{X}$ profile, which is compared with the 
368 corresponding best estimate and CI profiles obtained from each BCS interpretation. The 369 coverage proportion $\left(\mathrm{CP}_{\alpha}\right)$ of each of the 1000 RFSs that falls within the corresponding BCS $370 \mathrm{CI}_{\alpha}$ profiles was evaluated for different $\alpha$ values ranging from 50 to $95 \%$.

Figure 5a to 5c show histograms of $\mathrm{CP}_{50 \%}, \mathrm{CP}_{80 \%}$ and $\mathrm{CP}_{95 \%}$, respectively, when $\mathrm{M}=$ 37220 (i.e. $20 / 512=3.9 \%$ or less than $4 \%$ of the complete profile is measured). The mean $\mathrm{CP}_{50 \%}$, $373 \mathrm{CP}_{80 \%}$ and $\mathrm{CP}_{95 \%}$ values are shown in Figures 5a to $5 \mathrm{c}$ as $0.54,0.79$, and 0.92 , respectively.

374 These mean CP values are quite close to their respective $\alpha$ values (i.e. $0.5,0.8$ and 0.95 ,

375 respectively). Therefore, the confidence level may be interpreted as the expected coverage 376 proportion of the original and complete profile that falls within the corresponding BCS CI 377 profiles, if the original and complete profile can be measured. Additionally, similar to the 378 random field data discussed in the section "Coverage proportion of confidence interval 379 profiles", the CP probability distribution is close to symmetric when $\alpha=50 \%$ (see Figure $5 \mathrm{a}$ ). 380 As $\alpha$ increases and approaches unity, the distribution becomes less symmetric and presents a 381 negative skewness (see Figures $5 \mathrm{~b}$ and $5 \mathrm{c}$ ). Similar to Figure 2, Figure 6a shows box-and382 whiskers plots for $\mathrm{CP}_{\alpha}$ with various $\alpha$ values when $\mathrm{M}=20$. A 1:1 line is also included in 383 Figure 6a. All mean $\mathrm{CP}_{\alpha}$ values at various $\alpha$ levels plot close to the 1:1 line. This 384 demonstrates again that the confidence level may be interpreted as the expected coverage 385 proportion of the original and complete profile that falls within the corresponding CI profiles 386 obtained from BCS, if this original profile can be measured. Additionally, for relatively large $387 \alpha$ values, the data are negatively skewed, similar to the random field data shown in Figure $2 \mathrm{~b}$, 388 and the mean $\mathrm{CP}_{\alpha}$ value is slightly below $\alpha$. 
391 The BCS method was repeated with different number of measurement data points (M), 392 namely, $\mathrm{M}=10$ to 60 , with an increment of 10 points. These values correspond to 393 measurement spacing between $30 \mathrm{~cm}$ and $2 \mathrm{~m}$, and of fractions equal to $2 \%$ to about $12 \%$ of 394 the complete profile. As $M$ increases, more local variations of the original profile are 395 captured by the reconstructed BCS mean profile, as shown by Wang and Zhao (2017). 396 Additionally, as $\mathrm{M}$ increases, the bounds of the $\mathrm{CI}_{\alpha}$ profiles become narrow and approach to 397 the mean profile. This reflects that the statistical uncertainty is effectively reduced when more 398 data points are available. In this section, the effect of $M$ is evaluated on the coverage 399 proportion of the original and complete profile that falls within the corresponding CI profiles 400 obtained from BCS.

Figure 5 shows histograms of $\mathrm{CP}_{50 \%}, \mathrm{CP}_{80 \%}$ and $\mathrm{CP}_{95 \%}$ for three $\mathrm{M}$ values (i.e. $\mathrm{M}=20$, 40240 and 60). The mean $\mathrm{CP}_{50 \%}, \mathrm{CP}_{80 \%}$ and $\mathrm{CP}_{95 \%}$ values are shown in Figure $5 \mathrm{~d}$ to $5 \mathrm{f}$ for $\mathrm{M}=40$ 403 as $0.54,0.8$, and 0.93, respectively. In Figure $5 \mathrm{~g}$ to $5 \mathrm{i}$, the mean $\mathrm{CP}_{50 \%}, \mathrm{CP}_{80 \%}$ and $\mathrm{CP}_{95 \%}$ 404 values for $M=60$ are shown to be $0.48,0.77$, and 0.92 , respectively. For $M=20$ and $M=40$, the 405 mean $\mathrm{CP}_{50 \%}$ is slightly greater than $50 \%$ while the mean value for $\mathrm{CP}_{95 \%}$ is slightly less than $40695 \%$. Nonetheless, these mean $\mathrm{CP}_{\alpha}$ values are quite close to their respective $\alpha$ values. On 407 average a proportion $\alpha$ of all local variations of the original profile fall within the 408 corresponding $\mathrm{CI}_{\alpha}$ profiles, even when as few as $\mathrm{M}=20$ points are used to reconstruct the 409 mean and $\mathrm{CI}_{\alpha}$ profiles, which is less than $4 \%$ of the total data. In Figure 5 , it can be seen that 410 the variability of the $\mathrm{CP}_{\alpha}$ values decreases as $\mathrm{M}$ value increases. In addition, as previously 411 shown for $\mathrm{M}=20$ and for the full set of random field data, as $\alpha$ approaches unity the 412 distribution develops a negative skewness. However, as can be seen for $\mathrm{CP}_{80 \%}$ and $\mathrm{CP}_{95 \%}$, as 413 M increases, the distribution approaches to a normal distribution. 
414 Similar to Figure 6a, Figures $6 \mathrm{~b}$ and $6 \mathrm{c}$ show box-and-whiskers plots for $\mathrm{CP}_{\alpha}$ with 415 various $\alpha$ values when $M=40$ and 60 , respectively. 1:1 lines are also include in these figures. 416 As seen for $\mathrm{M}=20$ in Figure 6a, the mean $\mathrm{CP}_{\alpha}$ values at the $\alpha$ levels evaluated are close to the 417 1:1 line. In Figure 6 it is also evident that the variability of $\mathrm{CP}_{\alpha}$ values decreases as $\mathrm{M}$ 418 increases, e.g. see the size of the boxes in Figure 6. Figure 7 shows the mean values of $\mathrm{CP}_{50 \%}$, $419 \mathrm{CP}_{80 \%}$ and $\mathrm{CP}_{95 \%}$ for all values of $\mathrm{M}$ tested. Even though the statistical uncertainty is reduced 420 with increasing $\mathrm{M}$, the mean $\mathrm{CP}_{\alpha}$ is not greatly affected and fluctuates around the $\alpha$ value. 421 The relative difference between the average $\mathrm{CP}_{\alpha}$ and $\alpha$ is less than $15 \%$ in all the cases tested. 422 This demonstrates once again that the confidence level may be interpreted as the expected 423 coverage proportion of the original and complete profile that falls within the corresponding 424 BCS CI profiles.

\section{Effect of correlation length on $\boldsymbol{C P}_{\alpha}$}

427 To analyze the effect of the correlation length $\left(\lambda_{c}\right)$ on $\mathrm{CP}_{\alpha}$ results, new sets of RFSs are 428 generated using a truncated KL expansion with different $\lambda_{c}$. Eight $\lambda_{c}$ values were tested 429 ranging from 0.1 to $10 \mathrm{~m}$ to consider possible values of $\lambda_{c}$ for soil properties reported in the 430 literature (e.g. Phoon and Kulhawy 1999). For each RFS set, the BCS method was repeated 431 for three values of $\mathrm{M}$, namely $\mathrm{M}=20,40$ and 60 . Figure 8 shows the mean $\mathrm{CP}_{\alpha}$ results for all $432 \lambda_{c}$ and $\mathrm{M}$ values tested. When $\lambda_{c}$ is large (i.e. when there is a smoothly varying random 433 field), the average $\mathrm{CP}_{\alpha}$ tends to be greater than $\alpha$, indicating that the statistical uncertainty 434 reflected in the profiles for the bounds of $\mathrm{CI}_{\alpha}$ is greater than the variations with depth. In 435 other words, a relatively large proportion of many RFSs tested falls inside the corresponding $436 \mathrm{CI}_{\alpha}$ profiles. In contrast, with small values of $\lambda_{c}$, which implies a very variable field, the 
437 average $\mathrm{CP}_{\alpha}$ tends to be smaller than $\alpha$, indicating that a relatively small proportion of the 438 original profile falls inside the corresponding $\mathrm{CI}_{\alpha}$ bounds. The difference between the 439 average $\mathrm{CP}_{\alpha}$ and $\alpha$ decreases as $\mathrm{M}$ increases, irrespective of whether the $\lambda_{c}$ values used in the 440 simulation are small or large. For $\lambda_{c}$ values between $0.5 \mathrm{~m}$ and $2 \mathrm{~m}$, which are common values 441 for soil properties, the relative difference between the average $\mathrm{CP}_{\alpha}$ and $\alpha$ is less than $15 \%$.

442 The BCS method is robust and performs satisfactorily for the possible range of $\lambda_{c}$ values for 443 soil properties reported in the literature. It is worth noting that it is very difficult to determine 444 the correlation length $\left(\lambda_{c}\right)$ in engineering practice due to the limited measurement data. Using 445 the BCS method enables the need to determine the $\lambda_{c}$ value to be bypassed and provides the 446 best estimate and CI profiles for soil properties. In addition, note that, although an 447 exponential correlation function is used as illustrative examples in this paper, the method 448 proposed in the paper is general and equally applicable to other types of auto-correlation 449 function, and the BCS method performs well for other types of auto-correlation function.

\section{Illustrative example: Selection of effective friction angle profile}

452 In this section, the BCS method is demonstrated using a set of real CPT data for selection of 453 the characteristic value of the effective friction angle $\left(\phi^{\prime}\right)$. BCS provides the best estimate 454 profile of the effective friction angle and various CI profiles associated with various 455 confidence levels. These CI profiles may be used by engineers to facilitate determination of 456 the characteristic value profile in reliability-based design. To illustrate the proposed method, 457 only some of the normalized tip resistance $(q)$ values measured from CPT are used. First, the 458 BCS method is applied to provide the best estimate and CI profiles of $q$. The coverage 459 proportion of the BCS CI profiles is evaluated using the original and complete set of CPT 460 data. Then a transformation model is used to relate the $q$ profiles to $\phi^{\prime}$ profiles. The effect of 
461 the transformation model uncertainty on the $\phi^{\prime}$ profiles is also considered using Monte Carlo

462 simulations. Note that the $\phi^{\prime}$ profiles at various CI levels may be used by geotechnical

463 engineers to facilitate selection of the $\phi^{\prime}$ characteristic value in reliability-based design.

464 The CPT was performed on the Piedmont soils in Georgia Tech campus, Atlanta, in 465 which an extensive program of in-situ and laboratory tests has been carried out for soil 466 property determination (Mayne and Harris 1993). The BCS method is applied to the CPT 467 data in the residual silty sand layer between the depths of 3.8 and $19.2 \mathrm{~m}$, approximately 468 (Mayne and Harris 1993). Note that only one soil layer of residual silty sand is considered in 469 this illustrative example. Stratification therefore is not needed here. However, for a profile 470 with different soil layers, before application of the method proposed in this paper, 471 stratification of soil layers shall be performed, if possible, using, for example, Bayesian 472 method (Cao and Wang 2013; Wang et al. 2013,2014). In this layer, the cone tip resistance $473\left(q_{c}\right)$ ranges between $3.3 \mathrm{MPa}$ and $7.3 \mathrm{MPa}$, and the soil has a loose to medium-dense relative 474 density according to Meyerhof (1956). The soil has an average of $33 \%$ fines content, $8 \%$ 475 clays and a median grain size (D50) of $0.14 \mathrm{~mm}$. Note that although CPT data is used here for 476 illustration and validation, the BCS method really aims at the typical situation of sparsely 477 measured data (e.g. SPT data or laboratory test data) in engineering practice.

The cone tip resistance is normalized by the square root of the vertical effective stress ( $\left.\sigma_{v 0}^{\prime}\right)$ as follows: $q=\left(q_{c} / p_{a}\right) / \sqrt{\sigma_{v 0}^{\prime} / p_{a}}$, where $p_{a}$ is the atmospheric pressure. The 480 normalized tip resistance is shown in Figure 9a as a solid line. The BCS procedure is applied 481 to 15 data points (i.e. $M=15$ ), which represent a sampling interval of about $1 \mathrm{~m}$. These points 482 are also shown in Figure 9a as open circles. The best estimate and the bounds of the 90\% 483 confidence interval $\left(\mathrm{CI}_{90 \%}\right)$ obtained from BCS are shown in Figure 9a by a dashed and two 
484 dotted lines, respectively. The coverage proportion of the original CPT data within the $\mathrm{CI}_{90 \%}$ 485 profiles is $\mathrm{CP}_{90 \%}=85 \%$, which is close to the expected value of $90 \%$. and Mayne (1990) as follows:

$$
\phi^{\prime}=17.6+11 \log q
$$

Figure $9 \mathrm{~b}$ shows as a solid line the $\phi^{\prime}$ profile obtained when applying Equation (9) to the original and complete set of CPT data. Similarly, shown as dashed and two dotted lines are the results when applying Equation (9) to the profiles of the best estimate and the bounds of the $90 \%$ CI obtained from the BCS procedure. For comparison, Figures $9 \mathrm{~b}$ also includes lab test results from consolidated undrained triaxial compression tests that were performed using soil samples at different depths from this site (Mayne and Harris 1993). The mean $\phi^{\prime}$ from the 13 triaxial test data points is about $35^{\circ}$, which is similar to the value obtained from $\mathrm{BCS}$ (i.e. $35.39^{\circ}$ ). However, the triaxial data present more variability than that shown by the BCS $\mathrm{CI}_{90 \%}$ profiles.

Note that Equation (9) was obtained by a semi-log regression on twenty data sets from different sites, which cover site condition similar to that at Georgia Tech campus, Atlanta. A total of 633 data points was used in the regression. Significant residual error over Equation

501 (9) was reported, and the corresponding standard deviation of the residual error is $2.8^{\circ}$

502 (Kulhawy and Mayne 1990). This residual error can be treated as the model uncertainty of 503 Equation (9), and it may be included in Equation (9) as an additive zero-mean normally 
504 distributed random variable $\left(\varepsilon_{\mathrm{m}}\right)$. The $\varepsilon_{\mathrm{m}}$ in this example is modelled as a single random

505 variable to represent a perfectly correlated nature of $\varepsilon_{\mathrm{m}}$ over depth. To account for both the 506 model uncertainty and the BCS statistical uncertainty, Monte Carlo simulations were carried 507 out to provide the best estimate and various CI profiles for the effective friction angle. Five 508 thousand random samples of $\varepsilon_{\mathrm{m}}$ were generated in the simulations. Each $\varepsilon_{\mathrm{m}}$ sample was used 509 together with Equation (9), and a complete $q$ profile is reconstructed from BCS to generate a $510 \phi^{\prime}$ profile, leading to 5000 friction angle profiles. Then, the $\mathrm{CI}_{90 \%} \phi^{\prime}$ profiles with model 511 uncertainty was evaluated and shown in Figure 9c by two gray lines, together with those 512 without model uncertainty using the same symbols in Figure 9b. The interval given by the 513 two gray lines (i.e. with model uncertainty) is obviously much bigger than that given by two 514 dotted lines (i.e. without model uncertainty). It is obvious that the model uncertainty has 515 significant effect on the $\phi^{\prime}$ profiles. The $13 \phi^{\prime}$ data points from triaxial tests are also included 516 in Figure 9c. Eleven out of 13 data points (i.e. $11 / 13=85 \%$ ) fall within the $\mathrm{CI}_{90 \%} \phi^{\prime}$ profiles.

517 This is quite consistent with the statistical meaning of $\mathrm{CI}_{90 \%} \phi^{\prime}$ profiles that about $90 \%$ of 518 data points are expected to fall within the corresponding bounds. The lower bound of $\mathrm{CI}_{90 \%}$ 519 with both statistical and model uncertainty represents a $5 \%$ fractile of the $\phi^{\prime}$ profile and 520 might be selected as the characteristic value profile of $\phi^{\prime}$ in reliability-based design, if the 521 characteristic value is defined as the $5 \%$ fractile.

It is worth noting that the interpreted profile from the BCS method in this paper has 523 meaning similar to the local estimation of a geotechnical property of interest at the location 524 where a borehole was drilled (e.g. Honjo and Setiawan 2007; Honjo 2008). If global 525 estimation of a geotechnical property (i.e. the geotechnical property within the whole site) is 526 of interest (e.g. Honjo and Setiawan 2007; Honjo 2008), a perfect correlation in the 527 horizontal direction may be assumed. Alternatively, the BCS method may be extended from 
528 1D to 2D, and the perfect correlation assumption is not needed for 2D BCS method, which is

529 currently under development and beyond the scope of this study.

\section{Conclusions}

532 This paper developed a statistical procedure to facilitate objective selection of geotechnical 533 property characteristic value from spatially varying but sparsely measured data. The proposed 534 procedure is based on the Bayesian compressive sampling (BCS) method, which is not only 535 able to reconstruct the best estimate profile of a geotechnical property from sparse 536 measurement data, but also able to provide confidence interval (CI) profiles for quantifying 537 the statistical uncertainty associated with the interpretation. The quantified uncertainty in 538 BCS has a clear statistical meaning: the corresponding confidence level for BCS CI is the expected coverage proportion (i.e. fraction) of the complete profile that falls within the CI, if

540 all data points over the depth can be measured to provide the complete profile.

541 The statistical meaning of CI was firstly illustrated using random field data. When a

542 large number of complete sets of random field samples (RFSs) are used, the expected 543 coverage proportion $\left(\mathrm{CP}_{\alpha}\right)$ for a confidence interval with a confidence level $\alpha\left(\mathrm{CI}_{\alpha}\right)$ is equal 544 to $\alpha$. In addition, when only a limited number of data points from the RFS are measured, the 545 proposed $\mathrm{BCS}$ method can be used to reconstruct the best estimate and $\mathrm{CI}_{\alpha}$ profiles of the

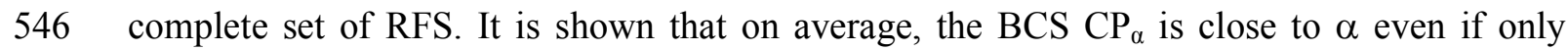
547 about $2 \%$ of the data points from the original and complete RFS are measured. As more data 548 points are available the statistical uncertainty is reduced and the variability in $\mathrm{CP}_{\alpha}$ also 549 reduces, but the average value is only slightly affected. In addition, the effect of the 550 correlation length $\left(\lambda_{c}\right)$ of the random field on the average $\mathrm{CP}_{\alpha}$ was also investigated. It is 
551 shown that the proposed method is robust and performs satisfactorily for the typical range of

$552 \lambda_{c}$ values for soil properties reported in the literature.

553 For geotechnical engineering applications, the complete set of data (i.e. a high-

554 resolution measurement data profile over depth) is often not available, and the BCS method

555 can be used to not only provide the best estimate profiles from sparse measurement data, but

556 also offer various confidence interval profiles. To illustrate this, BCS was used to estimate an

557 effective friction angle profile from sparse CPT data points in a real case history.

558 Furthermore, the uncertainty in the transformation model that relates CPT data to effective

559 friction angle can also be considered in the proposed method. It is shown that the effective

560 friction angle CI profiles from the proposed method using sparse CPT data points are

561 consistent with those from triaxial tests. Hence the best estimate and CI profiles from the

562 proposed method may be used to facilitate an objective determination of geotechnical

563 property characteristic values from sparse measurement data.

564

565 ACKNOWLEDGEMENT

566 The work described in this paper was supported by grants from the Research Grants Council

567 of the Hong Kong Special Administrative Region, China (Project No. 9042331 (CityU

568 11225216) and Project No. 8779012 (T22-603/15N)). The financial support is gratefully

569 acknowledged.

570

571

572 REFERENCES

573 AASHTO. 1998. LRFD bridge design specifications. Washington, D.C. 
574 Ang, A.H.-S., and Tang, W.H. 2007. Probability concepts in engineering: emphasis on 575 applications to civil and environmental engineering. New York: John Wiley and Sons.

576 Baecher, G.B., and Christian, J.T. 2003. Reliability and Statistics in Geotechnical 577 Engineering. Reliability and Statistics in Geotechnical Engineering. $578 \quad$ http://doi.org/10.1198/tech.2005.s838

Becker, D.E. 2010. Testing in geotechnical design. Geotechnical Engineering, 41(1).

Bond, A., and Harris, A. 2008. Decoding Eurocode 7. London and New York: Taylor \& 581

Candès, E.J., Romberg, J.K., and Tao, T. 2006. Stable signal recovery from incomplete and inaccurate measurements. Communications on Pure and Applied Mathematics, 59(8),

Candès, E.J., and Wakin, M.B. 2008. An Introduction To Compressive Sampling. IEEE Signal Processing Magazine, 25(2), 21-30. http://doi.org/10.1109/MSP.2007.914731

Cao, Z., and Wang, Y. 2013. Bayesian approach for probabilistic site characterization using cone penetration tests. Journal of Geotechnical and Geoenvironmental Engineering,

Cao, Z., and Wang, Y. 2014. Bayesian Model Comparison and Characterization of Undrained Shear Strength. Journal of Geotechnical and Geoenvironmental Engineering, 140(6).

Cao, Z., Wang, Y., and Li, D. 2016. Quantification of prior knowledge in geotechnical site 594 characterization. Engineering Geology, 203 107-116. http://doi.org/10.1016/j.enggeo.2015.08.018 
596 CEN 2004. Eurocode 7: Geotechnical design - Part 1: General rules, EN 1997-1:2004.

597 European Committee for Standardization (CEN), Brussels, Belgium.

598 CHDBC 2014. Canadian Highway Bridge Design Code. CAN/CSAS614:2014, Canadian 599 Standards Organization, Mississauga, Ontario, Canada.

600 Ching, J.Y. and Phoon, K.K. 2013a. Mobilized Shear Strength of Spatially Variable Soils $601 \quad$ Under Simple Stress States. Structural Safety, 41, 20-28.

602 Ching, J.Y. and Phoon, K.K. 2013b. Probability Distribution For Mobilized Shear Strengths 603 of Spatially Variable Soils Under Uniform Stress States. Georisk, 7(3), 209-224.

604 Ching, J.Y., Phoon, K.K. and Kao, P.H. 2014. Probability Model for Overall Shear Strength 605 of Spatially Variable Soils Under Uniform Stress States”, Journal of Engineering $606 \quad$ Mechanics, ASCE, 140(3), 487-501

607 Ching, J.Y., Hu, Y.G. and Phoon, K.K. 2016a. On Characterizing Spatially Variable Shear 608 Strength Using Spatial Average. Probabilistic Engineering Mechanics, 45, Jul 2016, 31$609 \quad 43$

610 Ching, J.Y., Lee, S.W. and Phoon, K.K. 2016b. Undrained Strength for a 3D Spatially 611 Variable Clay Column Subjected to Compression or Shear", Probabilistic Engineering $612 \quad$ Mechanics, 45, 127-139

613 Det Norske Veritas 2010. Statistical Representation of Soil Data. DNV-RP-C207.

614 De Veaux, R.D., Velleman, P.F., and Bock, D.E. 2014. Intro Stats (4th ed.). Pearson.

615 Efron, B., and Tibshirani, R.J. 1993. An Introduction to the Bootstrap. Chapman \& 616 Hall/CRC. Boca Raton, FL. http://doi.org/10.1111/1467-9639.00050 
617 Fenton, G.A., and Naghibi, M. 2011. Geotechnical resistance factors for ultimate limit state 618 design of deep foundations in frictional soils. Canadian Geotechnical Journal, 48(11), 619 1742-1756. https://doi.org/10.1139/t11-068

620 Fenton, G.A., and Griffiths, D.V. 2008. Risk Assessment in Geotechnical Engineering. Risk $621 \quad$ Assessment in Geotechnical Engineering. http://doi.org/10.1002/9780470284704

622 Foucart, S., and Rauhut, H. 2013. A Mathematical Introduction to Compressive Sensing. 623 Applied and Numerical Harmonic Analysis. http://doi.org/10.1007/978-0-8176-4948-7

624 Gong, W., Khoshnevisan, S., and Juang, C.H. 2014. Gradient-based design robustness 625 measure for robust geotechnical design. Canadian Geotechnical Journal, 51(11), 1331626 1342. doi: $10.1139 /$ cgj-2013-0428.

627 Ji, S., Xue, Y., and Carin, L. 2008. Bayesian compressive sensing. IEEE Transactions on 628 Signal Processing, 56(6), 2346-2356. http://doi.org/10.1109/TSP.2007.914345

629 Honjo, Y. and Setiawan B. 2007. General and local estimation of local average and their 630 application in geotechnical parameter estimations. Georisk, 1(3), 167-176. 631 http://dx.doi.org/10.1080/17499510701745960

632 Honjo, Y. 2008. General vs. local reliability based design in geotechnical engineering. In 633 Proceedings of the 4th Asian-Pacific Symposium on Structural Reliability and its 634 Applications (APSSRA'08), Hong Kong, June 19-20. pp. 41-52.

635 Kulhawy, F.H., and Mayne, P.W. 1990. Manual on Estimating Soil Properties for 636 Foundation Design. Ithaca, New York.

637 Li, D. Q., Shao, K.B., Cao, Z.J., Tang, X.S., and Phoon, K.K. 2016. A generalized surrogate 638 response aided-subset simulation approach for efficient geotechnical reliability-based 
88-101.

$640 \quad$ https://doi.org/10.1016/j.compgeo.2015.12.010

641 Li, D.Q., Qi, X.H., Phoon, K.K., Zhang, L.M., and Zhou, C.B. 2014. Effect of spatially 642 variable shear strength parameters with linearly increasing mean trend on reliability of 643 infinite slopes. Structural Safety, 49, 45-55. doi:

644 http://dx.doi.org/10.1016/j.strusafe.2013.08.005.

645 Marra, G., and Wood, S.N. 2012. Coverage Properties of Confidence Intervals for 646 Generalized Additive Model Components. Scandinavian Journal of Statistics, 39(1), 53647 74. http://doi.org/10.1111/j.1467-9469.2011.00760.x

648 Mathworks. 2016. MATLAB - The language of technical computing.

649 Mayne, P.W., and Harris, D.E. 1993. Axial load-displacement behavior of drilled shaft $650 \quad$ foundations in piedmont residuum. McLeam, Virginia.

651 Meyerhof, G.G. 1956. Penetration tests and bearing capacity of cohesionless soils. Journal of 652 the Soil Mechanics and Foundations Division, ASCE, 82(SM1), 1-19.

653 Meyerhof, G.G. 1995. Development of geotechnical limit state design. Canadian 654 Geotechnical Journal, 32(1), 128-136. http://doi.org/10.1139/t95-010

655 Nychka, D. 1988. Bayesian confidence intervals for smoothing splines. Journal of the 656 American Statistical Association, $\quad 83(404), \quad 1134-1143$. 657 http://doi.org/10.1080/01621459.1988.10478711 
659 Orr, T.L.L. 2017. Defining and selecting characteristic values of geotechnical parameters for 660 designs to Eurocode 7. Georisk: Assessment and Management of Risk for Engineered 661 662 $\begin{array}{lll}\text { Systems } \quad \text { and } \quad \text { Geohazards, } & 11(1),\end{array}$ http://doi.org/10.1080/17499518.2016.1235711

663

664

665

666

667

668

669

670

671

672

673

674

675

676

677

678

679
Phoon, K.-K., and Ching, J. 2014. Risk and Reliability in Geotechnical Engineering. Boca Raton, FL: CRC Press, Taylor \& Francis Group.

Phoon, K.-K., Huang, H.W., and Quek, S.T. 2005. Simulation of strongly non-Gaussian processes using Karhunen-Loeve expansion. Probabilistic Engineering Mechanics, 20(2), 188-198. http://doi.org/10.1016/j.probengmech.2005.05.007

Phoon, K.-K., Huang, S.P., and Quek, S.T. 2002. Implementation of Karhunen-Loeve expansion for simulation using a wavelet-Galerkin scheme. Probabilistic Engineering Mechanics, 17(3), 293-303. http://doi.org/10.1016/S0266-8920(02)00013-9

Phoon, K.-K., and Kulhawy, F.H. 1999. Characterization of geotechnical variability. Canadian Geotechnical Journal, 36(4), 612-624. http://doi.org/10.1139/t99-038

Phoon, K.-K., Prakoso, W. A., Wang, Y., and Ching, J. 2016. Chapter 3 Uncertainty representation of geotechnical design parameters. In Reliability Of Geotechnical Structures In Iso2394 (pp. 49-88). http://doi.org/10.1201/9781315364179-4

Reddy, S.C., and Stuedlein, A.W. 2017. Ultimate Limit State Reliability-Based Design of Augered Cast-in-Place Pile Considering Lower-Bound Capacities. Canadian Geotechnical Journal, doi: 10.1139/cgj-2016-0145. 
680 Schneider, H.R., and Schneider, M.A. 2013. Dealing with uncertainties in EC7 with emphasis 681 on determination of characteristic soil properties. Modern Geotechnical Design Codes of 682 Practice, P. Arnold et al. (Eds.), ISO Press, 87-101.

683 Taboga, M. 2012. Lectures on Probability Theory and Mathematical Statistics (Second Edi). 684 CreateSpace Independent Publishing Platform.

685 Vanmarcke, E.H. 1977. Probabilistic Modeling of Soil Profiles. Journal of the Geotechnical 686 Engineering Division, 103(11), 1227-1246.

Vick, S. 2002. Degrees of Belief - Subjective Probability and Engineering Judgment, ASCE $688 \quad$ Press.

Wahba, G. 1983. Bayesian "Confidence Intervals" for the Cross-validated Smooth Spline. Journal of the Royal Statistical Society. Series B: Statistical Methodology, 45(1), 133-

Wang, Y., Akeju, O.V., and Cao, Z. 2016a. Bayesian Equivalent Sample Toolkit (BEST): an 150 Excel VBA program for probabilistic characterisation of geotechnical properties from limited observation data. Georisk: Assessment and Management of Risk for Engineered Systems and Geohazards, 10(4), 251-268.

Wang, Y., and Aladejare, A.E. 2015. Selection of site-specific regression model for characterization of uniaxial compressive strength of rock. International Journal of Rock Mechanics \& Mining Sciences, 75, 73-81. Strength Index at a specific site. Rock Mechanics and Rock Engineering, 49(9), 35593573. 
702 Wang, Y., and Cao, Z. 2013. Probabilistic characterization of Young's modulus of soil using 703 704 http://doi.org/10.1016/j.enggeo.2013.03.017 equivalent $\quad$ samples. Engineering Geology, 159, 106-118.

Wang, Y., Cao, Z., and Li, D. 2016b. Bayesian perspective on geotechnical variability and site characterization. Engineering Geology, 203, 117-125. http://doi.org/10.1016/j.enggeo.2015.08.017

Wang, Y., and Zhao, T. 2016. Interpretation of soil property profile from limited measurement data: a compressive sampling perspective, Canadian Geotechnical Journal, 53(9), 1547-1559. http://doi.org/10.1139/cgj-2015-0545

711 Wang, Y., Huang, K., and Cao, Z. 2013. Probabilistic identification of underground soil 712 stratification using cone penetration tests. Canadian Geotechnical Journal, 50(7), 766713776.

714 Wang, Y., Huang, K., and Cao, Z. 2014. Bayesian identification of soil strata in London clay. $715 \quad$ Géotechnique, 64(3), 239.

716 Wang, Y., and Zhao, T. 2017. Statistical interpretation of soil property profiles from sparse 717 data using Bayesian compressive sampling. Geotechnique, 718 http://dx.doi.org/10.1680/jgeot.16.P.143.

719 Zhang, J., and Ellingwood, B. 1994. Orthogonal Series Expansions of Random Fields in 720 Reliability Analysis. Journal of Engineering Mechanicsg, 120(12), 2660-2667. 
721 Figure Captions

722 Figure 1: $\mathrm{N}_{\mathrm{s}}=1000$ sets of random field samples (RFSs) generated for soil property X (The 723 95\% coverage proportion $\left(\mathrm{CP}_{95 \%}\right)$ for the three RFS examples is indicated as title of each 724 subplot)

725 Figure 2: Box-and-whiskers plot for the coverage proportion $\left(\mathrm{CP}_{\alpha}\right)$ as a function of the 726 confidence level $(\alpha)$ for different correlation length $\left(\lambda_{c}\right):$ (a) $0.5 \mathrm{~m}$, (b) $2 \mathrm{~m}$ and (c) $5 \mathrm{~m}$ (Mean 727 values are shown with circles; the median values are shown with a line inside the box; and 728 the minimum and maximum values are shown with crosses)

729 Figure 3: Effect of the correlation length $\left(\lambda_{c}\right)$ on $\mathrm{CP}_{\alpha}$ : (a) Box plots for $\mathrm{CP}_{50 \%}, \mathrm{CP}_{80 \%}$ and

$730 \mathrm{CP}_{95 \%}$; (b) Standard deviation of $\mathrm{CP}_{\alpha}\left(\sigma_{\mathrm{CP} \alpha}\right)$ as a function of $\alpha$ (The analytical solution for the 731 case with no correlation (i.e. $\lambda_{c}=0$ ) is shown with a solid line)

732 Figure 4: Three simulated $\mathrm{X}$ profiles and those reconstructed from $\mathrm{BCS}$ using $\mathrm{M}=20$ 733 measurement data points $y$

734 Figure 5: Histograms of the coverage proportion $\left(\mathrm{CP}_{\alpha}\right)$ for $50 \%, 80 \%$ and $95 \%$ confidence 735 levels (The red vertical lines show the confidence level)

736 Figure 6: Box-and-whiskers plot for the coverage proportion $\left(\mathrm{CP}_{\alpha}\right)$ of the original RFS profile 737 within the given BCS CI under different M scenarios: (a) $M=20$, (b) $M=40$ and (c) $M=60$

738 Figure 7: Effect of the number of measurement data points $(\mathrm{M})$ on the mean $\mathrm{CP}_{\alpha}$ values for 739 confidence levels $50 \%, 80 \%$ and $95 \%$.

$740 \quad$ Figure 8: Effect of the correlation length $\left(\lambda_{c}\right)$ on the mean $\mathrm{CP}_{\alpha}$ values 
741 Figure 9: Results of illustrative example: estimation of effective friction angle $\phi^{\prime}$ from

742 normalized CPT tip resistance $q$ : (a) Comparison between the original $q$ profile and the $q$

743 profile reconstructed from $\mathrm{BCS}$ (b) Best estimate and $\mathrm{CI}_{90 \%}$ profiles of $\phi^{\prime}$ without

744 consideration of model uncertainty $\varepsilon_{\mathrm{m}}$ (c) Best estimate and $\mathrm{CI}_{90 \%}$ profiles of $\phi^{\prime}$ with

745 consideration of model uncertainty $\varepsilon_{\mathrm{m}}$

746 

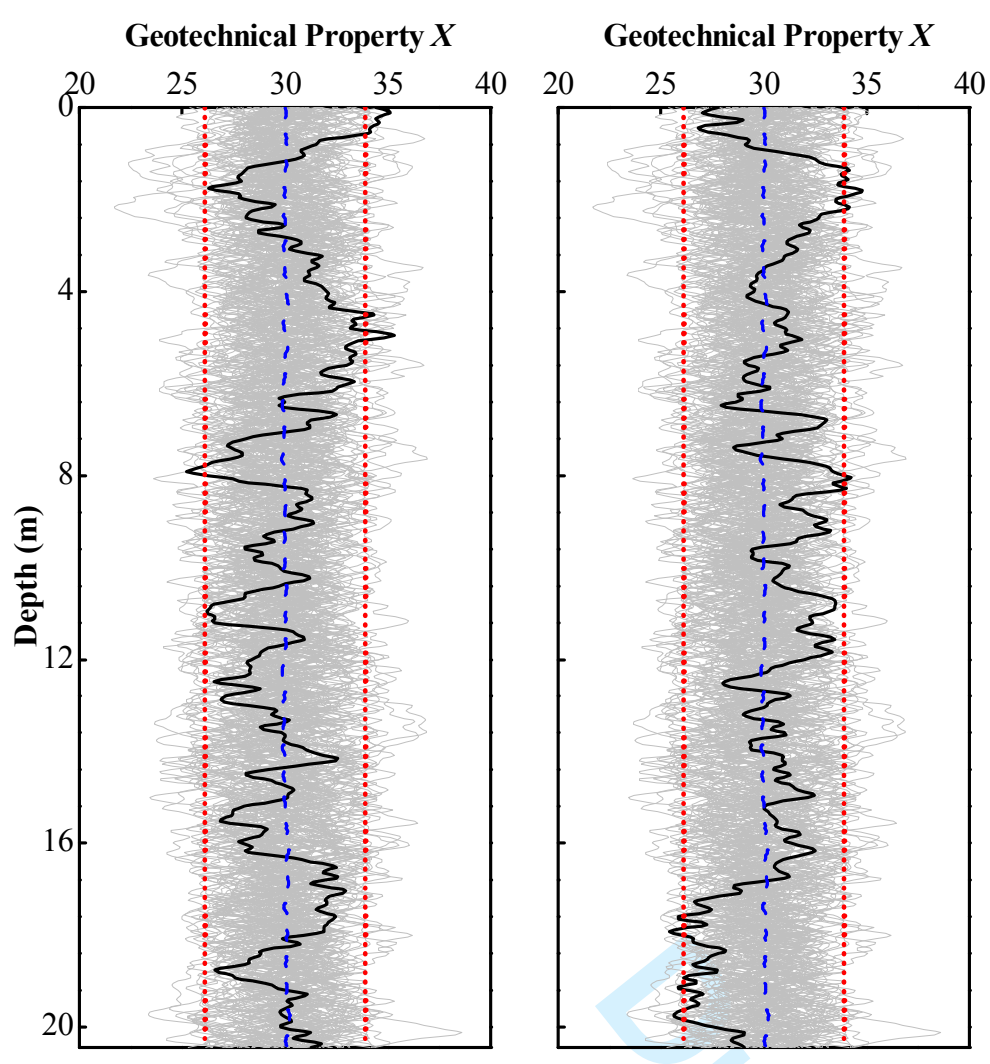

Geotechnical Property $X$

1

$\mathrm{N}_{\mathrm{s}}$ simulated $X$ profiles

Examples of $X$ profiles

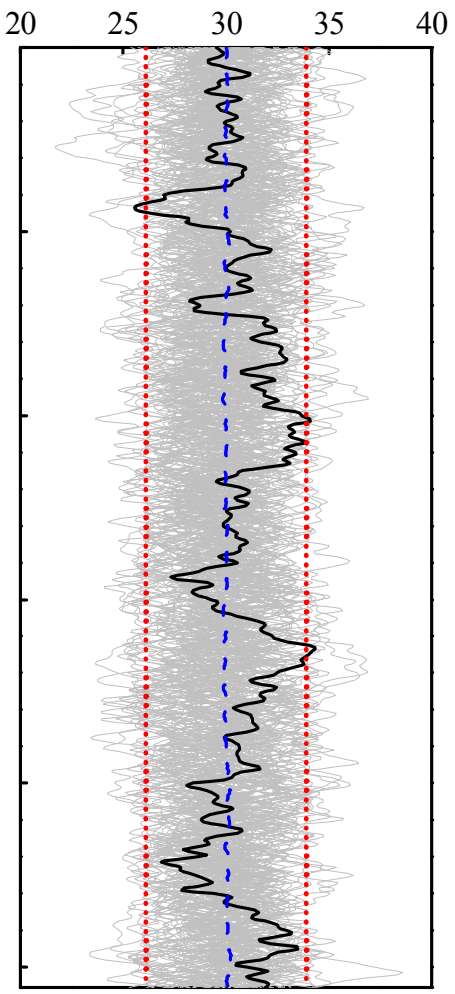
(a) $\mathrm{CP}_{95 \%}=94.5 \%$
(b) $\mathrm{CP}_{95 \%}=94.9 \%$
(c) $\mathrm{CP}_{95 \%}=96.1 \%$

2 Figure 1: $\mathrm{N}_{\mathrm{s}}=1000$ sets of random field samples (RFSs) generated for soil property $\mathrm{X}$ (The

$395 \%$ coverage proportion $\left(\mathrm{CP}_{95 \%}\right)$ for the three RFS examples is indicated as title of each

4 subplot) 
6

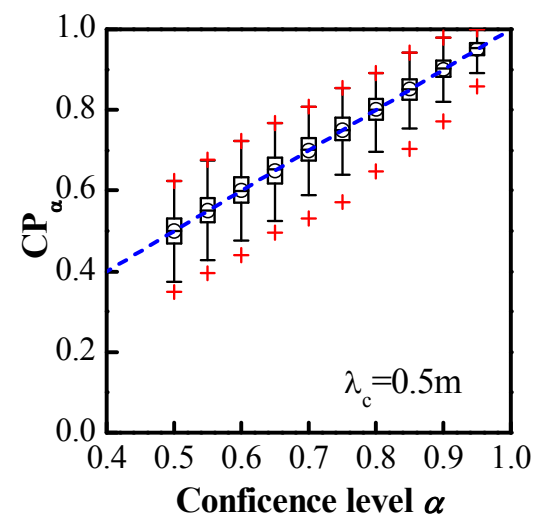

(a)

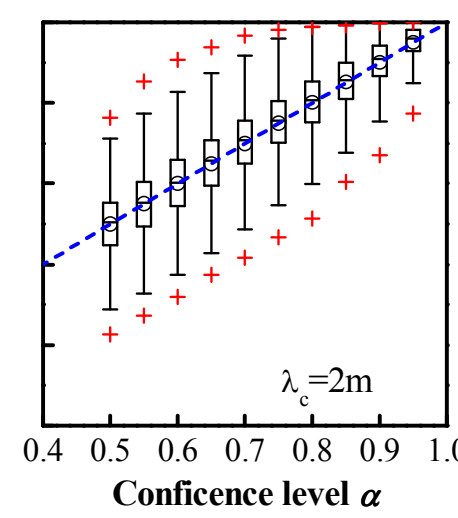

(b)

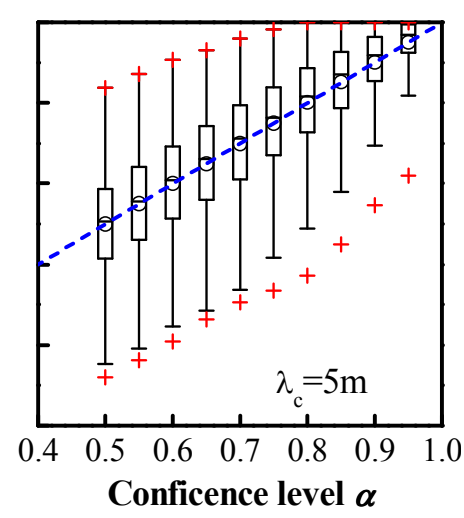

(c)

8 Figure 2: Box-and-whiskers plot for the coverage proportion $\left(\mathrm{CP}_{\alpha}\right)$ as a function of the

9 confidence level $(\alpha)$ for different correlation length $\left(\lambda_{c}\right)$ : (a) $0.5 \mathrm{~m}$, (b) $2 \mathrm{~m}$ and (c) $5 \mathrm{~m}$ (Mean

10 values are shown with circles; the median values are shown with a line inside the box; and

11 the minimum and maximum values are shown with crosses) 


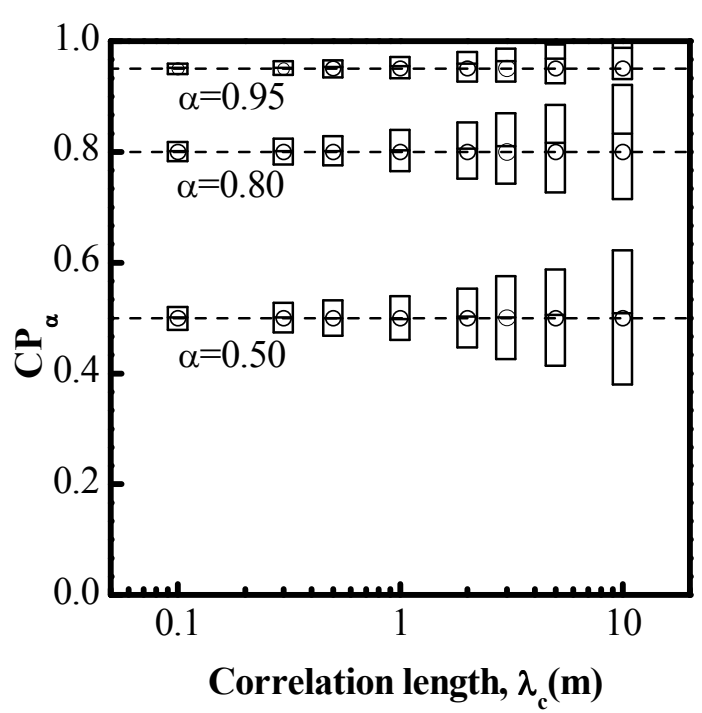

(a)

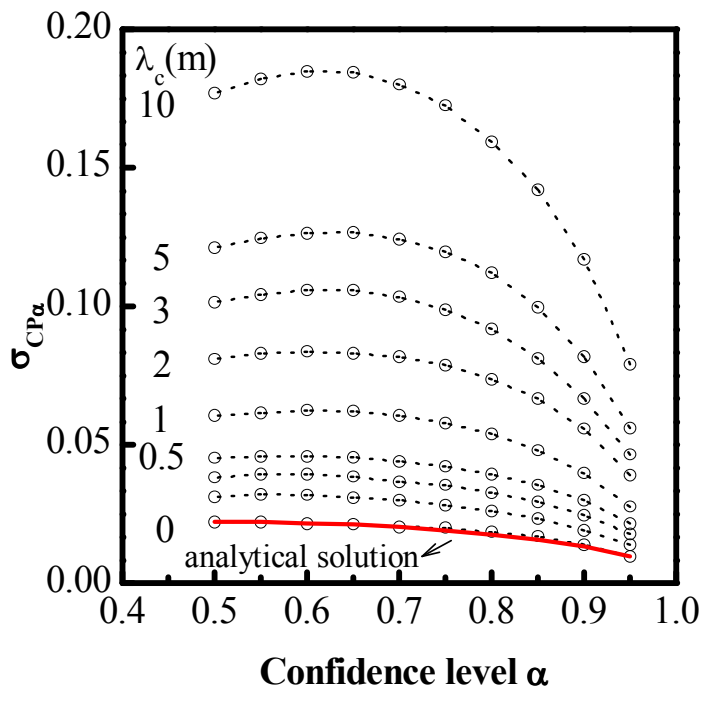

(b)

14 Figure 3: Effect of the correlation length $\left(\lambda_{c}\right)$ on $\mathrm{CP}_{\alpha}$ : (a) Box plots for $\mathrm{CP}_{50 \%}, \mathrm{CP}_{80 \%}$ and

$15 \mathrm{CP}_{95 \%}$; (b) Standard deviation of $\mathrm{CP}_{\alpha}\left(\sigma_{\mathrm{CP} \alpha}\right)$ as a function of $\alpha$ (The analytical solution for the

16 case with no correlation (i.e. $\lambda_{c}=0$ ) is shown with a solid line)

17 


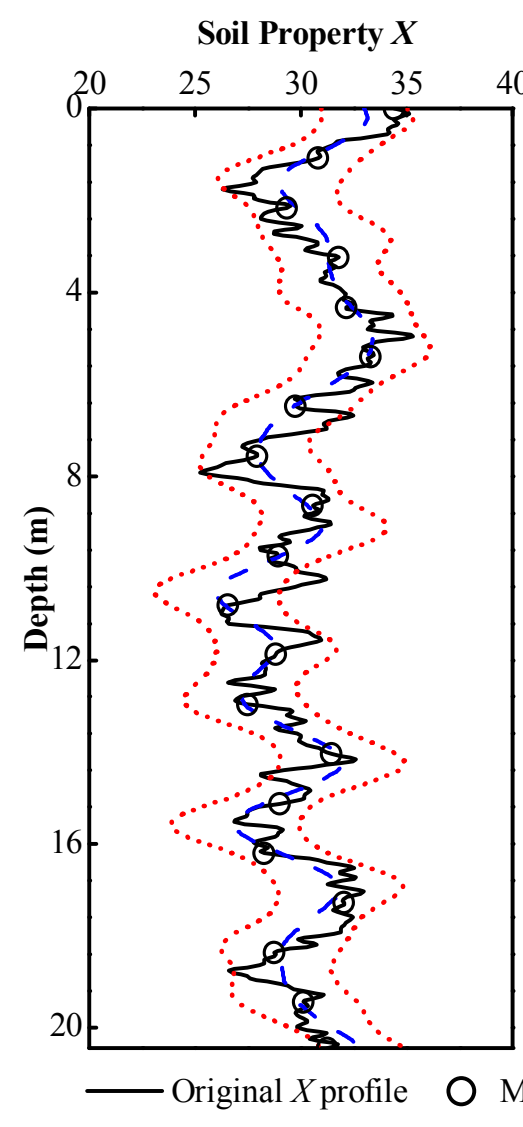

Soil Property $\boldsymbol{X}$

Soil Property $X$

$40 \quad 20$

$\begin{array}{lll}25 & 30 \quad 35\end{array}$

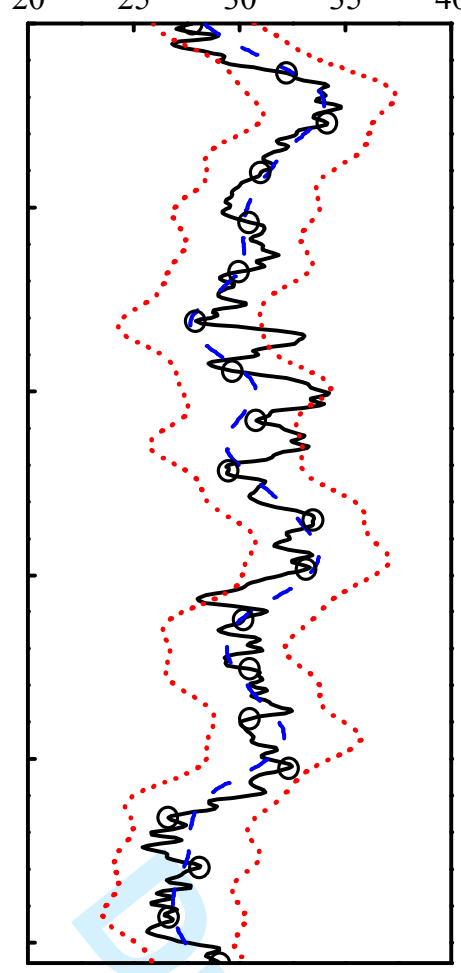

$\begin{array}{llllll}40 & 20 & 25 & 30 & 35 & 40\end{array}$

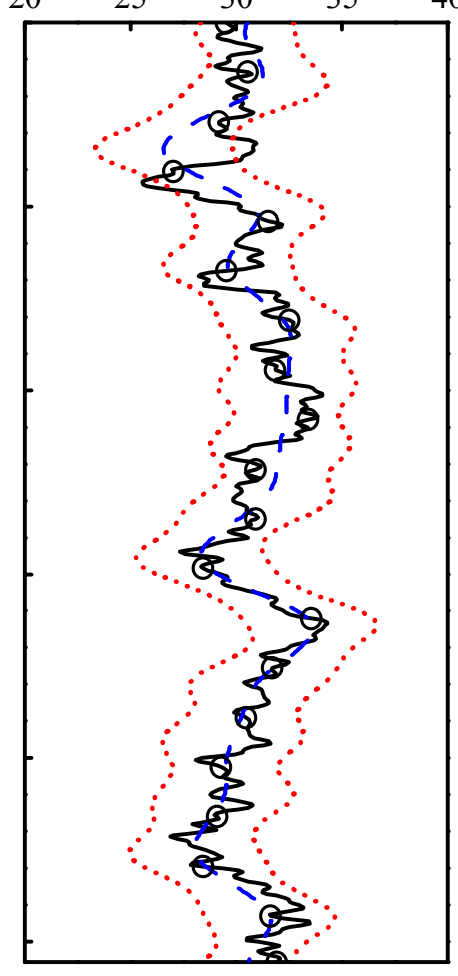

19
(a) $\mathrm{CP}_{95 \%}=94.9 \%$
(b) $\mathrm{CP}_{95 \%}=94.1 \%$
(c) $\mathrm{CP}_{95 \%}=96.3 \%$

20 Figure 4: Three simulated $\mathrm{X}$ profiles and those reconstructed from BCS using $\mathrm{M}=20$

21 measurement data points $\boldsymbol{y}$ 

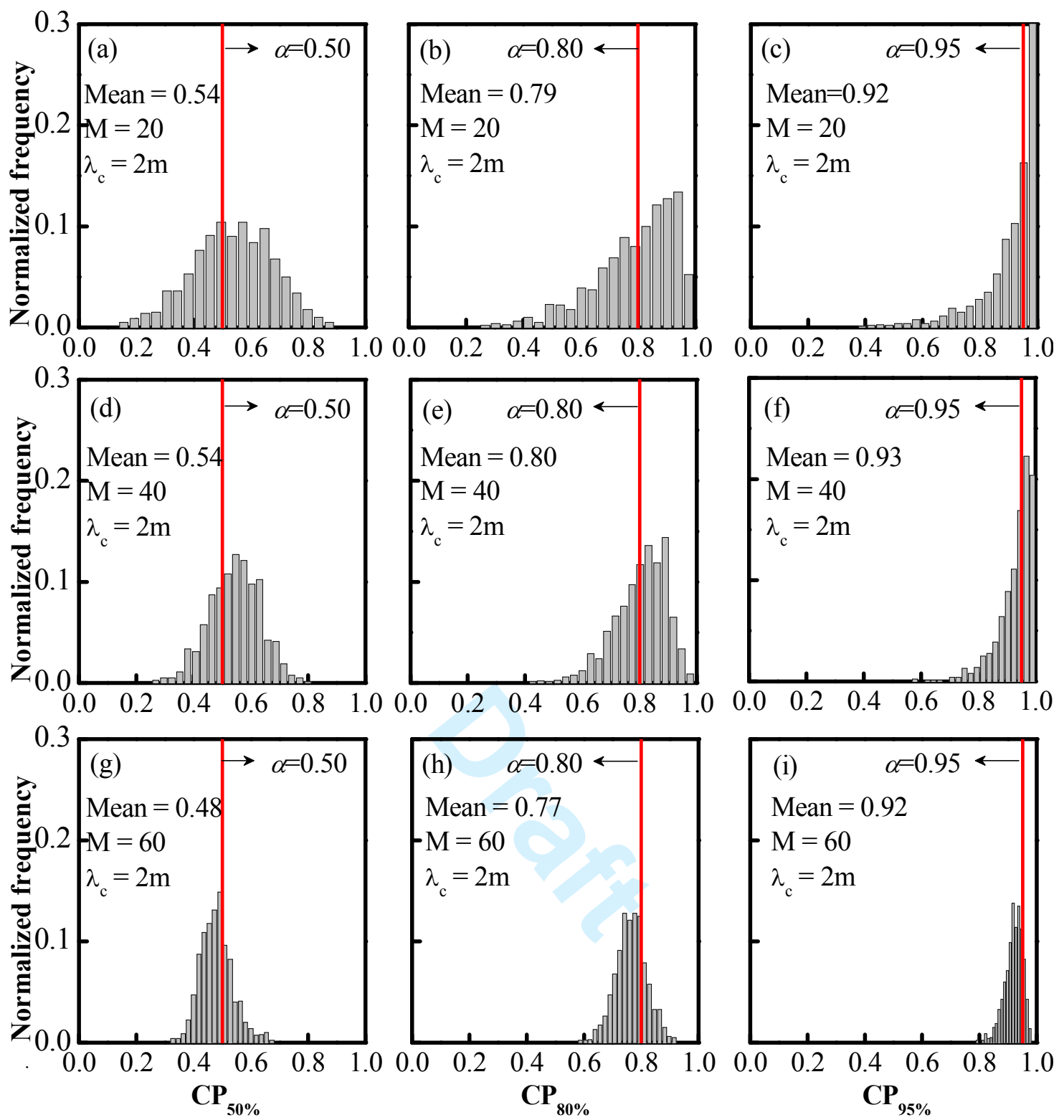

24 Figure 5: Histograms of the coverage proportion $\left(\mathrm{CP}_{\alpha}\right)$ for $50 \%, 80 \%$ and $95 \%$ confidence

25 levels (The red vertical lines show the confidence level) 


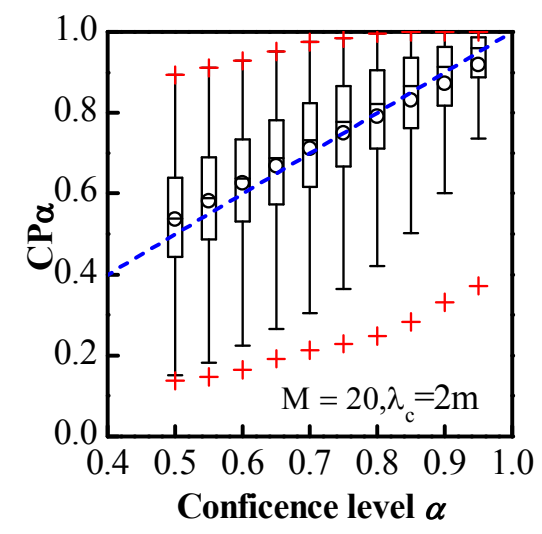

(a)

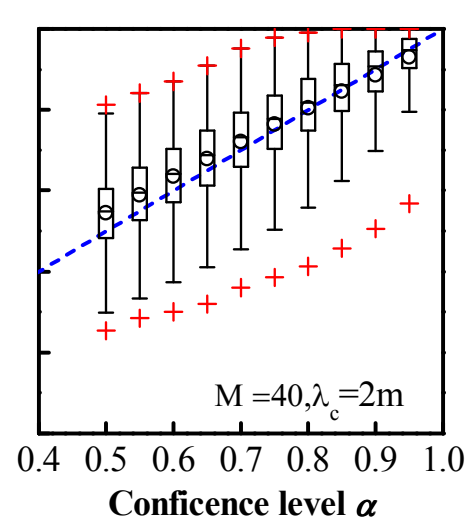

(b)

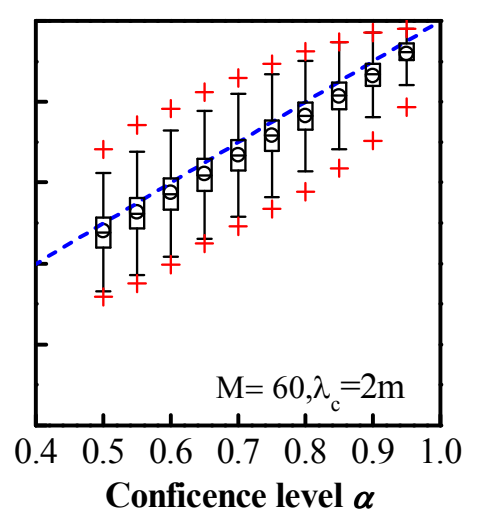

(c)

28 Figure 6: Box-and-whiskers plot for the coverage proportion $\left(\mathrm{CP}_{\alpha}\right)$ of the original RFS profile within the given BCS CI under different M scenarios: (a) $M=20$, (b) $M=40$ and (c) $M=60$ 


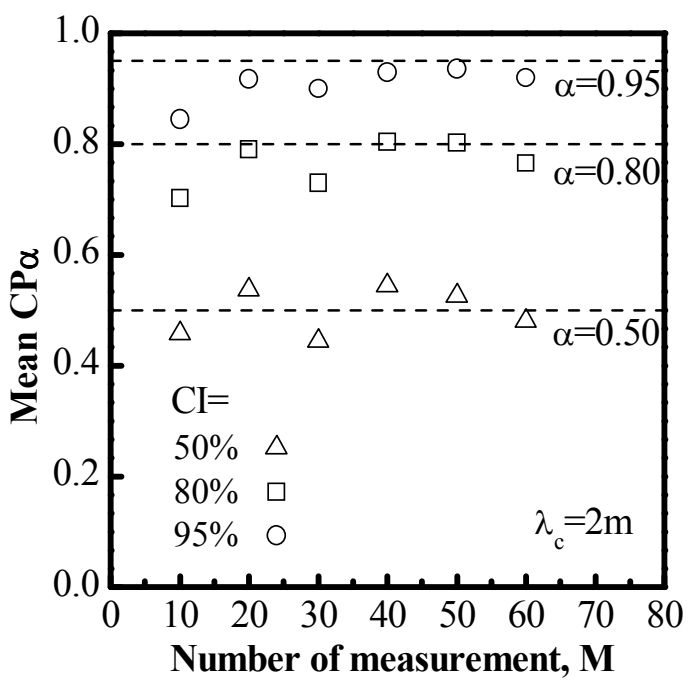

31

32 Figure 7: Effect of the number of measurement data points (M) on the mean $\mathrm{CP}_{\alpha}$ values for 33 confidence levels $50 \%, 80 \%$ and $95 \%$. 


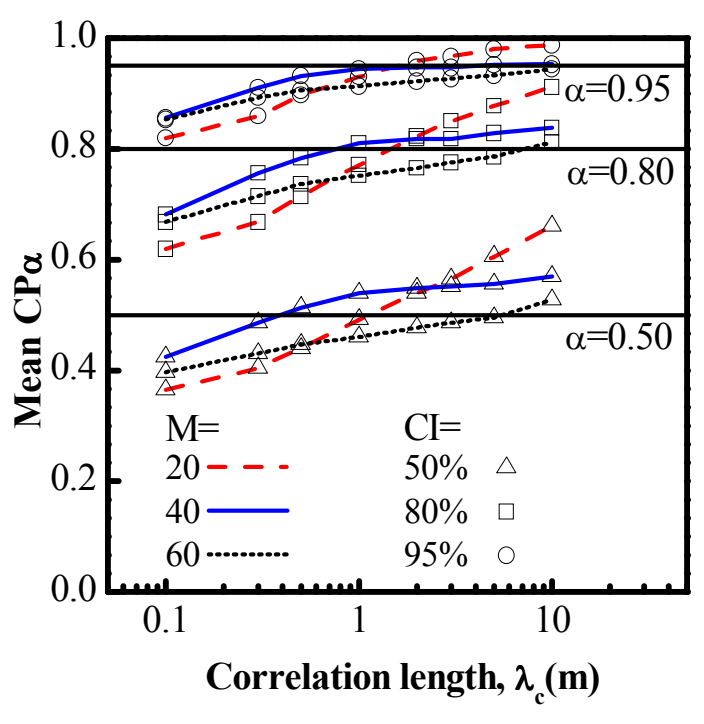

35

36 Figure 8: Effect of the correlation length $\left(\lambda_{c}\right)$ on the mean $\mathrm{CP}_{\alpha}$ values 


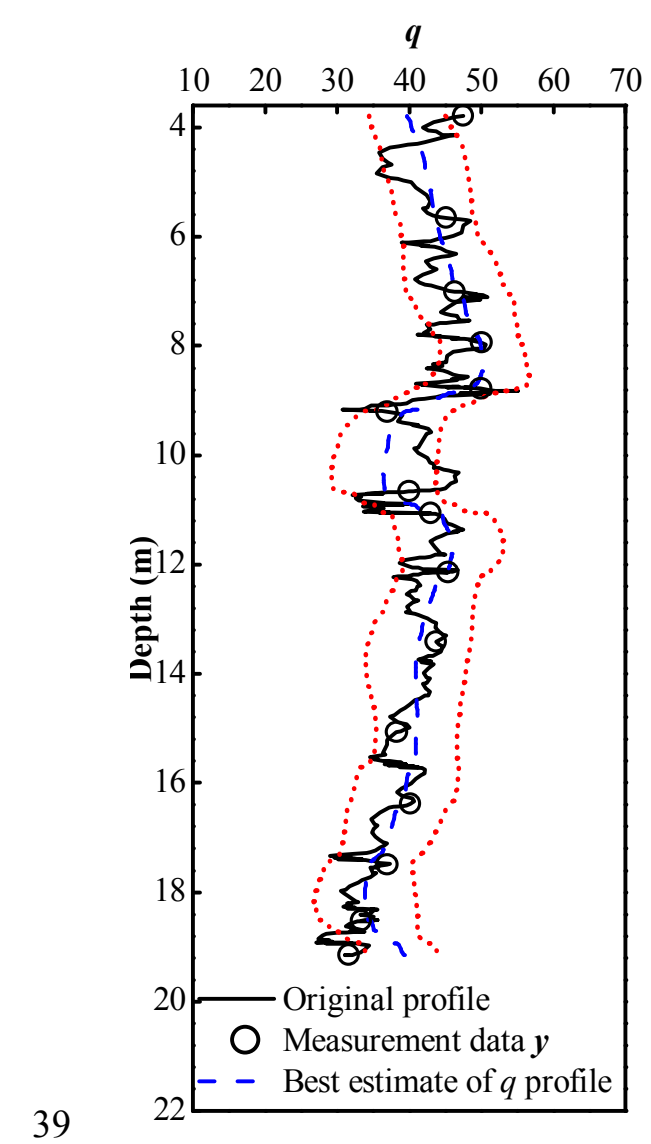

(a)

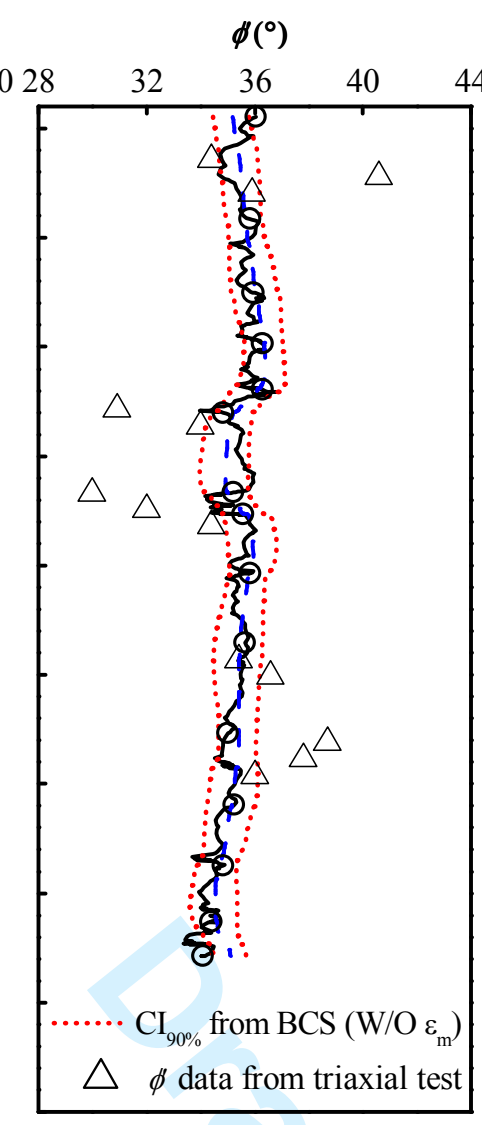

(b)

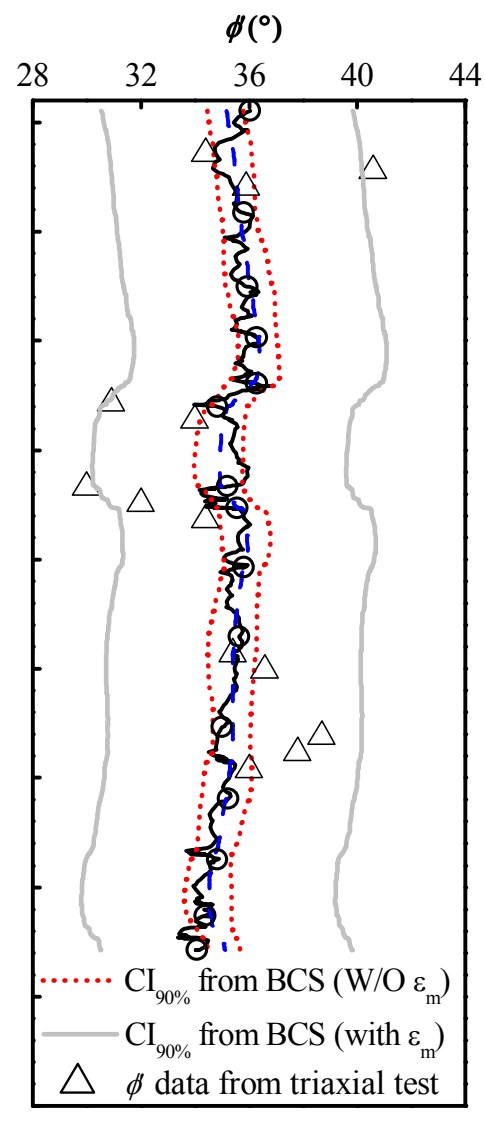

(c)

40 Figure 9: Results of illustrative example: estimation of effective friction angle $\phi^{\prime}$ from

41 normalized CPT tip resistance $q$ : (a) Comparison between the original $q$ profile and the $q$

42 profile reconstructed from $\mathrm{BCS}$ (b) Best estimate and $\mathrm{CI}_{90 \%}$ profiles of $\phi^{\prime}$ without

43 consideration of model uncertainty $\varepsilon_{\mathrm{m}}$ (c) Best estimate and $\mathrm{CI}_{90 \%}$ profiles of $\phi^{\prime}$ with

44 consideration of model uncertainty $\varepsilon_{\mathrm{m}}$ 